\title{
Volcanic plume simulation on large scales
}

\author{
Josef M. Oberhuber ${ }^{\text {a, * }}$, Michael Herzog ${ }^{\text {b }}$, Hans-F. Graf ${ }^{\text {b }}$, Karsten Schwanke ${ }^{\text {b }}$ \\ a German Climate Computing Centre, Model Development and Application Group, Bundesstraße 55, 20146 Hamburg, Germany \\ b Max-Planck Institute for Meteorology, Bundesstraße 55, 20146 Hamburg, Germany
}

Received 5 January 1998; accepted 6 August 1998

\begin{abstract}
The plume model ATHAM (Active Tracer High Resolution Atmospheric Model) is designed to simulate explosive volcanic eruptions for a given mass flux of pyroclastic material under realistic atmospheric background conditions. Based on the assumption that all particles are small the model's equations are simplified such that, besides equations for gaseous, liquid and solid constituents of arbitrary concentrations, only the volume means of momentum and heat are predicted. The exchange of momentum and heat between the fluid's constituents are treated diagnostically. A prognostic turbulence closure scheme describing the entrainment of ambient air into the plume takes into account the anisotropy of the horizontal and vertical components of turbulence. Its length scale is assumed to be isotropic. Microphysical processes such as the exchange of heat and momentum between dry air, water vapor, cloud water, precipitable water, ice crystals and graupel are parameterized. Ash and lapilli represent the spectrum of silicate particles. A diagnostic sedimentation velocity allows for the separation of gas and particles. The model is formulated with an implicit time stepping scheme. The equations of motion and the transport equations for tracers are formulated in flux form in order to guarantee the conservation of momentum and all tracer masses. The heat transport equation is in advective form. The wave equation and the equations for the transport of momentum, heat and tracers are solved using a combined line-relaxation successive overrelaxation scheme. Two-dimensional experiments for symmetric cases with cylindrical coordinates yield qualitatively similar results to other dynamic-thermodynamic models. However, entrainment processes are now computed quantitatively through the turbulence closure and condensed matter has a sophisticated description. In order to study the transferability of results from computationally cheap two-dimensional experiments to costly three-dimensional simulations of a realistic plume, comparisons between calculations with and without cylindrical coordinates are performed. Finally, experiments for different atmospheric background conditions allow investigation of plume development on the influence of cross wind effects, and temperature and humidity profiles. (C) 1998 Elsevier Science B.V. All rights reserved.
\end{abstract}

Keywords: volcanic plume; ATHAM; simulation

\section{Introduction}

As global atmospheric models are increasingly efficient in simulating the global circulation including the stratosphere in detail, there are increasing

\footnotetext{
* Corresponding author
}

activities to model the atmospheric chemistry. In particular, the stratosphere is of interest because of long residence times of some of the chemical species. For instance, chlorine and bromine have a strong capacity to destroy ozone. Others like sulfate, besides their effect on heterogenous chemistry, can change the circulation in the stratosphere by affect- 
ing the radiation budget. One of the important natural sources of these chemicals are volcanos, either in phases of quiet degassing or during a single volcanic eruption. So far, chemical reactions and solution processes in volcanic plume water phases are not yet fully understood. However, these processes are needed to determine the vertical profile used in large-scale atmospheric modelling. The modelling of this input profile needs to take $\mathrm{H}_{2} \mathrm{O}$ into account as a further volcanic gas because it is important for the dynamics of a volcanic plume due to latent heat release during condensation and sublimation to cloud water and ice. Water phases are also capable of dissolving chlorine or sulfate, which might be carried with the plume up into the stratosphere. While some volcanic gases may reside in the atmosphere for years, small silicate particles, also called ash, settle within days to a month depending on whether an ash cloud is washed out quickly within the troposphere or succeeds to penetrate into the stratosphere. Larger silicate particles such as lapilli or bombs are of importance in successful modelling of the lower part of the plume and for building up sediment layers, which may be used to relate plume properties with sediment distribution and thus help to interpret sediments left from historical volcanic eruptions.

One-dimensional plume models (e.g., Woods, 1988) describe the steady state solution of a plume under idealized boundary conditions. Specific aspects of a plume, such as height, are considered by Settle (1978) and Wilson et al. (1978). The competition between velocity loss and buoyancy gain through entrainment is discussed by Sparks and Wilson (1976) and Bursik and Woods (1991). Even though determination of essential parameters such as the entrainment rate are not based on first principles such as a turbulent energy budget, such simple models nevertheless allow us to investigate the importance of physical mechanisms in a volcanic plume. Other studies like those of Woods (1993) and Glaze et al. (1997) demonstrate that condensation/deposition of water vapor becomes important already during the first minutes of an eruption. This is a strong indication that microphysical processes cannot be neglected on longer time scales. Such simple models can only give qualitative answers as microphysical processes are rather crudely parameterized and feedbacks with the dynamics are not well-resolved.
An early effort to model the dynamics of the initial minutes of an eruption was made by Wohletz et al. (1984) focusing on the blast wave phenomenon. More recently, multi-component models such as those of Valentine and Wohletz (1989), Dobran et al. (1993) and Neri and Macedonio (1996) describe the dynamics and thermodynamics in such detail that their models can be considered as examples of multiphase flow models. These models henceforth are called universal models. In this model concept each component is described by a complete set of prognostic equations for momentum, mass and heat. The interaction between these components such as the exchange of momentum and heat is treated prognostically. Such concepts, although computationally expensive when used for dozen of components, allow description of non-equilibrium processes between the various phases with high resolution in time and space.

The aim of this study is to develop a model that provides the link between the generation of a plume at the surface and the input of chemical species into the stratosphere. The model should resolve spatial scales of a plume that are typical for the near-vent area up to scales of several hundred kilometers horizontally, and at least $50 \mathrm{~km}$ height. One of the key quantities to be simulated is the altitude to which the plume rises. This determines the input of water, ashes and chemical species into the stratosphere. To simulate the rising plume and the interaction with the atmospheric background state correctly, the model must be transient and three-dimensional, although qualitative studies will be possible in two dimensions. Finally, the model should be cheap enough to be able to extend integrations until the simulated state becomes stationary after a volcanic eruption, which is several hours. After extracting profiles of horizontal averages, they can be fed into a regional or finer-resolution large-scale atmospheric general circulation model in order to compute tracer transport and related chemistry. To achieve this, the model needs to solve the equations of motion and all those additional quantities that are of relevance for chemical reactions and dissolution processes of gases into cloud droplets. These are the dynamic and thermodynamic quantities, and the environmental conditions such as the presence of the wet and ice phase of $\mathrm{H}_{2} \mathrm{O}$. However, for the sake of computational 
efficiency, approximations need to be made in order to perform experiments with reasonable spatial resolution over hours of duration despite considering a big number of solid and liquid particle classes.

The paper is structured as follows. In Section 2, the model is described, starting with the model concept (Section 2.1), followed by a discussion of the equations for the dynamics, the thermodynamics, and the tracers (Section 2.2). The model equations are derived in Section 2.3. In Section 2.4, the turbulence closure scheme is summarized. In Section 2.5, the interface to the microphysic module is described. In Section 2.6, the atmospheric background state is described, and finally, in Section 2.7, the numerical techniques are discussed. Section 3 presents a description of the experimental setups (Section 3.1), selected results for idealized eruptions with and without cylindrical coordinates (Section 3.2), and followed by a discussion of experiments with realistic background conditions (Section 3.3). Section 4 summarizes the paper.

\section{Model description}

The model to be presented is designed to describe the injection of a mixture of hot gases and particles of various properties into the atmosphere under realistic lower and lateral boundary conditions. Besides the simulation of the fluid's laminar flow, the role of turbulence is essential for simulating a volcanic plume. This is because turbulence induces a net transport between fluids of highly different properties. This is called entrainment, which, for instance, may increase the buoyancy of a plume when ambient air is mixed into the particle-laden hot plume. However, these mixing processes may also be suppressed in situations of strong vertical stratification such as those occurring at the interface between pyroclastic flows and the ambient air. If time scales for the development and decay of a plume are considered, then cloud microphysics needs to be included, i.e., the conversion of heat mainly due to condensation of water vapor and conversion between cloud droplets, rain drops and various forms of ice. This is important as the ultimate goal of the model is to serve as a carrier of chemical species, and to simulate the environmental conditions that control chemical reactions and dissolution processes of gases. The model must therefore be capable of considering compressible flow and dynamic/thermodynamic interaction between all constituents. Furthermore, realistic parameterizations for the effect of turbulence and microphysical processes must be developed.

\subsection{The model concept}

In order to develop an affordable model, the exchange of momentum and heat between the fluid's constituents is assumed to be in equilibrium at all times. To do this we solve only one equation for the volume mean momentum, and one for the volume mean heat content as opposed to three equations for the velocity components and one for heat content for each tracer in universal models. This assumption means that the adjustment time scales of the exchange processes are proportionally faster than the model's time-resolution. The exchange processes for momentum and heat then are treated diagnostically rather than prognostically. However, compared with universal models this gain in computational efficiency is balanced by spending computer-resources on a bigger number of tracers as, for instance, required to model the microphysics and chemistry of a volcanic plume. Because each additional tracer results in only one additional prognostic equation for a scalar quantity as opposed to four in a universal model, the computing time does not dramatically increase with an increase in the number of tracers.

\subsubsection{Definitions}

The volume mean of a quantity is the sum of the mass weighted contribution from all constituents of the fluid. If $q_{n}$ denotes the specific concentration of the $n$th tracer $X_{n}$, then the volume mean quantity $X$ is defined as $X=\sum_{n} q_{n} X_{n}$ with $\sum_{n} q_{n}=1$. In this sense scalar quantities without subscript or vector quantities like the velocity $u_{i}$ with no further subscript denote volume mean quantities, while a subscript $n$ denotes some tracer or alternatively the subscript $g$ denotes the gas phase.

An equilibrium is characterized by zero net fluxes between constituents of the fluid. Generally, liquid 
and solid particles have a velocity relative to the gas, called slip velocity. If the drag of the particles with the ambient air is in balance with the gravitational force, then this is called dynamic equilibrium. In respect to temperature, thermodynamic equilibrium means that all constituents of the fluid have the same in situ temperature.

\subsubsection{Dynamic equilibrium}

Due to friction with the ambient air the velocity difference between particles and gas eventually reaches a constant value. Under the influence of the earth's gravity this velocity stays at a finite non-zero value for the vertical component and causes sedimentation of particles. It mostly depends on the size, shape, density and environmental conditions like gas density. Unlike the vertical and because of the absence of an external force in the horizontal plane, the horizontal component of the velocity difference falls to zero. If particles are assumed to be small, then the limiting factor is the time needed for a particle to pass a grid cell vs. the time needed to approach a stationary velocity relative to the surrounding gas. For small particles such as the liquid/solid water phase and ash, the stationary slip velocity is only a few meters per second. Thus the gravitational force together with an adjustment time of a few seconds results in a critical grid resolution of a few decameters. Larger particles like juvenile lapilli usually have a low density due to the high concentration of gas bubbles they contain. Thus, the stationary sedimentation velocity for these particles is less than $10 \mathrm{~m} / \mathrm{s}$ (Walker et al., 1971). As for bombs and blocks, and smaller lithic particles, a dynamic equilibrium is never achieved, the presented model concept cannot consider them. It may be concluded that a resolution of $100 \mathrm{~m}$ near the vent is sufficiently coarse in order not to clearly violate the assumption of dynamic equilibrium. It is therefore sufficient to predict the volume mean momentum. This is equivalent to the statement that the net pressure force on each fluid's component is identical to the volume mean pressure force, which can be expressed by:

$p=p_{\mathrm{g}}$

where $p$ is the volume mean pressure required for the prediction of the volume mean momentum and $p_{\mathrm{g}}$ is the gas pressure later required by the equation of state for gas.

\subsubsection{Thermodynamic equilibrium}

The second approximation is that the volume mean in situ temperature $T$ is identical to the in situ temperature $T_{n}$ of each component:

$T=T_{n}$.

This implies that the heat conductivity is efficient enough to transfer heat quickly from the core of a particle to its surface and finally to the surrounding gas. This simplification is valid only for small particles. As found by Woods and Bursik (1991) and Neri and Macedonio (1996), a significant thermal disequilibrium may already occur for typical lapilli diameters. This is because in cases of strong accelerations such as occurring in pyroclastic flows, slip velocities may considerably exceed their stationary fallout velocities. Then heat conductivity is not efficient enough to remove temperature differences between gas and particles before a particle has left a specific grid cell within a model time step. In a future version of the model a compromise might be worth considering, namely to add a prognostic heat equation for bigger particles. Then the heat exchange between these few classes of bigger particles and the remainder of the fluid can be parameterized in the same way as in fully prognostic models.

\subsection{The model equations}

As discussed so far, the concept is to predict volume mean for momentum, heat and pressure and predict the specific concentration of each tracer. The interaction between the fluid's constituents is treated diagnostically. Further diagnostic relations between individual densities and concentrations of the fluid's constituents, temperature and pressure with volume mean density complete the set of equations. A list of variables is given in Table 1 . 
Table 1

List of model variables. If a subscript $g$ is used instead of $n$ then the gas component is considered rather than an arbitrary constituent of the fluid

\begin{tabular}{|c|c|c|}
\hline Variable & Unit & Meaning \\
\hline$u_{1}, u_{2}, u_{3}$ & $\mathrm{~ms}^{-1}$ & volume mean velocity in $x$-, $y$ - and $z$-direction \\
\hline$u_{1, n}, u_{2, n}, u_{3, n}$ & $\mathrm{~ms}^{-1}$ & velocity in $x-, y$ - and $z$-direction of $n$th component \\
\hline$\rho$ & $\mathrm{kg} \mathrm{m}^{-3}$ & volume mean density \\
\hline$\rho_{n}$ & $\mathrm{~kg} \mathrm{~m}^{-3}$ & density of $n$th component \\
\hline$p, p_{\mathrm{g}}$ & $\mathrm{N} \mathrm{m}^{-2}$ & volume mean pressure, gas pressure \\
\hline$\Theta$ & $\mathrm{K}$ & volume mean potential temperature \\
\hline$T$ & $\mathrm{~K}$ & volume mean in situ temperature \\
\hline$T_{n}$ & $\mathrm{~K}$ & in situ temperature of $n$th component \\
\hline$R_{\mathrm{g}}$ & $\mathrm{m}^{2} \mathrm{~s}^{-2} \mathrm{~K}^{-1}$ & gas constant \\
\hline$c_{\mathrm{p}}^{\circ}$ & $\mathrm{m}^{2} \mathrm{~s}^{-2} \mathrm{~K}^{-1}$ & specific heat capacity for constant pressure \\
\hline$Q^{\Theta}$ & $\mathrm{K} \mathrm{s}^{-1}$ & heat forcing \\
\hline$K^{\mathrm{m}}, K^{\mathrm{q}}, K^{\Theta}$ & $\mathrm{m}^{2} \mathrm{~s}^{-1}$ & diffusion coefficient for momentum, tracers and heat \\
\hline$B_{\text {hor }}$ & $\mathrm{m}^{2} \mathrm{~s}^{-2}$ & horizontal turbulent kinetic energy component \\
\hline$B_{\text {ver }}$ & $\mathrm{m}^{2} \mathrm{~s}^{-2}$ & vertical turbulent kinetic energy component \\
\hline$B$ & $\mathrm{~m}^{2} \mathrm{~s}^{-2}$ & total turbulent kinetic energy \\
\hline$L$ & $\mathrm{~m}$ & turbulent length scale \\
\hline$L_{0}$ & $\mathrm{~m}$ & turbulent equilibrium length scale \\
\hline$N$ & $\mathrm{~s}^{-1}$ & Brunt-Vaisalla frequency \\
\hline$c_{0}, c_{1}, c_{2}, c_{3}$ & & empirical constants for turbulence closure \\
\hline$c_{\mathrm{s}}$ & $\mathrm{ms}^{-1}$ & speed of sound \\
\hline$\alpha_{\Theta}, \alpha_{q}, \alpha_{\sigma}$ & & Prandtl number for heat, tracers and turbulence \\
\hline
\end{tabular}

\subsubsection{The dynamics}

The equations of motion in component form and the continuity equation can be written:

$$
\begin{aligned}
\frac{\partial}{\partial t} \rho u_{i}= & -\frac{\partial}{\partial x_{j}} u_{j} \rho u_{i}+\frac{\partial}{\partial x_{j}} \rho K_{j}^{m} \frac{\partial}{\partial x_{j}} u_{i}-\frac{\partial}{\partial x_{i}} p \\
& -\rho\left(g \delta_{i 3}-2 \epsilon_{i j k} \omega_{j}\left(u_{k}-u_{k}^{*}\right)\right)+Q_{i}^{m} \\
\frac{\partial}{\partial t} \rho q_{g}= & -\frac{\partial}{\partial x_{i}} \rho q_{g} u_{i, g}+Q^{g}
\end{aligned}
$$

where $\rho$ is the volume mean density, $u_{i}$ the volume mean velocity vector, $q_{g}$ the specific gas concentration, $K_{j}^{m}$ the turbulent exchange coefficient for momentum (see Section 2.4), $p$ the volume mean pressure, $g$ the gravitational constant, $\delta_{i j}$ is Dirac's delta function, $Q_{i}^{m}$ is the external force due to input of momentum at the vent and $Q^{g}$ is the sum of mass input of gas through the vent and sources/sinks of mass due to phase change processes. The Coriolis force enters via an atmospheric background wind profile prescribed by $u_{k}^{*}$ and the earth's angular velocity $\omega_{j}$ which is defined as:

$\omega_{j}=\Omega(0, \cos \varphi, \sin \varphi)$ with $\Omega=\frac{2 \pi}{86164}$

The $\epsilon$-tensor is used to represent the vector product through the definition:

$$
\begin{aligned}
& \boldsymbol{\epsilon}_{i, j, k} \\
& \quad=\left\{\begin{aligned}
1 & \text { if }(i, j, k) \text { even permutation of }(1,2,3) \\
-1 & \text { if }(i, j, k) \text { odd permutation of }(1,2,3) \\
0 & \text { otherwise. }
\end{aligned}\right.
\end{aligned}
$$


The vertical velocity component for gas $u_{3, g}$ required for the continuity equation (Eq. (4)) for the gas mass is defined through the definition of the predicted volume mean flow and all slip velocities relative to the gas:

$$
\left(u_{1, g}, u_{2, g}, u_{3, g}\right)=\left(u_{1}, u_{2}, u_{3}-\sum_{n}^{n \neq g}\left(q_{n} \Delta u_{3, n}\right)\right)
$$

The slip velocity $\Delta u_{3, n}$ is defined in the microphysics module (see Herzog et al., 1998) while the horizontal gas velocities $u_{1, g}$ and $u_{2, g}$ are by definition equal to the volume mean velocities due to the neglect on horizontal slip velocities.

\subsubsection{The tracer equation}

Tracers are constituents of the fluid that are carried with the flow. Often, tracers are understood as constituents of the fluid that do not directly alter the dynamics via the equation of state. In our context these are called passive tracers. In contrast, tracers in the present model are called active as they are allowed to have such a high concentration that they cannot be neglected in the equation of state for the volume mean density. Therefore the model is called Active Tracer, and because the model has a high resolution when compared with global models, has the extension High resolution Atmosphere Model. The prognostic equation in flux form reads:

$$
\frac{\partial}{\partial t} \rho q_{n}=-\frac{\partial}{\partial x_{i}} q_{n} \rho u_{i, n}+\frac{\partial}{\partial x_{i}} K_{i}^{q} \frac{\partial}{\partial x_{i}} \rho q_{n}+Q_{n}^{q}
$$

where $q_{n}$ is the specific concentration of the $n$th tracer and $Q_{n}^{q}$ is its external forcing due to mass input through the vent and mass conversion due to microphysical processes. $K_{i}^{q}$ is the turbulent exchange coefficient (see Section 2.4). The fallout velocities relative to the surrounding gas $\Delta u_{3, n}$ for the $n$th tracer together with the predicted volume mean velocity $u_{i}$ are used to separate the individual velocities for each tracer in order to obtain $u_{\mathrm{i}, n}$ :

$\left(u_{1, n}, u_{2, n}, u_{3, n}\right)=\left(u_{1}, u_{2}, u_{3, g}+\Delta u_{3, n}\right)$

Because the fallout velocities are due to gravity, the horizontal components of the flow are identical for all tracers.
All experiments discussed in Section 3 of this paper use water vapor, ash, lapilli, cloud water, precipitable water, ice crystals and graupel as tracers. The specific concentration of dry gas is implicitly defined through $\sum_{n} q_{n}=1$. The specific concentration of gas is the sum of the predicted specific concentration of water vapor and the diagnostically computed specific concentration of dry gas (see Section 2.2.4).

\subsubsection{The thermodynamics}

The prediction of the temperature distribution is carried out in terms of an advection-diffusion equation for the volume mean potential temperature $\Theta$, where the item advection is used as a synonym for laminar transport and the item diffusion for turbulent transport or exchange processes. In contrast to the potential temperature of gas $\Theta_{g}$, which is not conservative because gas may exchange heat with other constituents of the fluid, the volume mean potential temperature is a conservative quantity if defined as:

$$
\Theta=\frac{\sum_{n}\left(q_{n} c_{p, n} \Theta_{n}\right)}{\sum_{n}\left(q_{n} c_{p, n}\right)}
$$

where $\Theta_{n}$ are the individual potential temperatures of gas or tracers (see Section 2.2.4). Then the prognostic equation for $\Theta$ in advective form is:

$$
\frac{\partial}{\partial t} \Theta=-u_{i, \Theta} \frac{\partial}{\partial x_{i}} \Theta+\frac{1}{\rho} \frac{\partial}{\partial x_{i}} \rho K_{i}^{\Theta} \frac{\partial}{\partial x_{i}} \Theta+Q^{\Theta}
$$

where $K_{i}^{\Theta}$ is the turbulent exchange coefficient for heat (see Section 2.4) and $Q^{\Theta}$ the heat forcing due to microphysical processes and due to input of heat through the vent. The vertical transport of $\Theta$ requires the use of an effective vertical velocity $u_{3, \Theta}$ in order to consider the differential efficiency of each component to transport heat at a given velocity by weighing each component with its individual specific heat capacity and specific concentration. Eq. (11) can be derived from the aforementioned universal models by merging together all the prognostic equations for heat. The relation for the effective transport velocity $u_{i, \Theta}$ for $\Theta$ is:

$$
\left(u_{1, \Theta}, u_{2, \Theta}, u_{3, \Theta}\right)=\left(u_{1}, u_{2}, \frac{\sum_{n}\left(q_{n} c_{p, n} u_{3, n}\right)}{\sum_{n}\left(q_{n} c_{p, n}\right)}\right)
$$


where $u_{3, n}$ is defined in Eq. (9). A definition of an effective horizontal transport velocity is not necessary as all individual horizontal velocity components are identical.

\subsubsection{Equation of state}

In order to relate the in situ gas temperature $T_{g}$, gas pressure $p_{g}$ and gas density $\rho_{g}$ the ideal gas equation is used:

$p_{g}=R_{g} \rho_{g} T_{g}$ with $R_{g}=c_{p, g}-c_{v, g}$

where $R_{g}$ is the gas constant, and $c_{p, g}$ and $c_{v, g}$ are the specific heat capacities for constant pressure and volume. The gas potential temperature $\Theta_{g}$ is related with the in situ gas temperature $T_{g}$ by:

$T_{g}=\Theta_{g}\left(\frac{p_{g}}{p_{0}}\right)^{\frac{c_{p, g}-c_{v, g}}{c_{p, g}}}$

where $p_{0}$ is a common reference pressure. Note again that $T_{n}=\Theta_{n}$ for all liquid and solid constituents. As gas is allowed to consist of several constituents with different specific heat capacities, these are defined as mass weighted sum over all gases:

$c_{p, g}=\frac{\sum_{n}^{n=g} q_{n} c_{p, n}}{\sum_{n}^{n=g} q_{n}}$ and $c_{v, g}=\frac{\sum_{n}^{n=g} q_{n} c_{v, n}}{\sum_{n}^{n=g} q_{n}}$

where $\sum_{n}^{n=g}$ is understood as sum over all gas constituents only. In this paper only dry air and water vapor are used. Starting with the definition of the total heat content through Eq. (10) and taking into account that the in situ temperatures $T_{n}$ are identical for all liquid and solid particles the in situ temperature $T$ is obtained from:

$$
T=\frac{\Theta \sum_{n} q_{n} c_{p, n}}{\left(\frac{p_{g}}{p_{0}}\right)^{\frac{R_{g}}{c_{p}}} c_{p, g} q_{g}+\sum_{n}^{n \neq g} c_{p, n} q_{n}} .
$$

Because the densities of liquid and solid constituents are assumed to be incompressible, i.e., they are constants, and the gas density can be computed using
Eq. (13), the volume mean density can be determined from:

$$
\frac{1}{\rho}=\sum_{n} \frac{q_{n}}{\rho_{n}} .
$$

\subsection{Concept of a prognostic pressure equation}

Explosive volcanic eruptions are characterized by flows that can easily exceed the speed of sound. In addition, the turbulence closure scheme will result in big turbulent exchange coefficients, i.e., long diffusive time scales near locations of strong turbulent energy production which, if treated with explicit techniques, would result in very small time steps. An implicit time step scheme is therefore chosen in order to save computing time, especially in periods of weak winds before and after an eruption, and strong turbulence. An appropriate strategy is to derive a prognostic pressure equation and formulate the resulting elliptic equation implicitly in terms of a system of linear equations (see Section 2.7.2).

Starting with the total differential for the gas pressure and using Eqs. (13) and (14), the time derivative of the gas pressure can be related to that of the gas density and the gas potential temperature:

$$
\frac{\partial}{\partial t} p_{g}=\frac{c_{p, g}}{c_{v, g}} p_{g}\left(\frac{1}{\rho_{g}} \frac{\partial}{\partial t} \rho_{g}+\frac{1}{\Theta_{g}} \frac{\partial}{\partial t} \Theta_{g}\right)
$$

The problem now is that the continuity equation for gas (see Eq. (4)) predicts $\rho q_{g}$ while a prognostic equation is needed for $\rho_{g}$. After rewriting the left hand side of Eq. (4) the continuity equation for gas becomes:

$$
\frac{\partial}{\partial t} \rho_{g}=-\frac{\rho_{g}}{\rho q_{g}}\left(\frac{\partial}{\partial x_{i}} q_{g} \rho u_{i, g}-Q^{q}+\rho_{g} \frac{\partial}{\partial t} \frac{\rho q_{g}}{\rho_{g}}\right)
$$

where the last term on the right-hand side represents the time-derivative of the gas-covered volume, which is a small correction and is obtained from:

$$
\frac{V}{V_{g}}=\frac{m}{\rho} \frac{\rho_{g}}{m_{g}}=\frac{\rho_{g}}{\rho q_{g}}
$$


where $V$ is the volume of a grid cell with its total mass content $m$ and $V_{g}$ that part of it that is occupied by gas only with $m_{g}$ its gas mass contents. Finally, the gas pressure tendency equation is:

$$
\begin{aligned}
\frac{\partial}{\partial t} p_{g}= & \frac{c_{p, g}}{c_{v, g}}\left(\frac{p_{g}}{\Theta_{g}} \frac{\partial}{\partial t} \Theta_{g}-\frac{p_{g}}{\rho q_{g}}\left(\frac{\partial}{\partial x_{i}} \rho q_{g} u_{i, g}-Q^{q}\right.\right. \\
& \left.\left.+\rho_{g} \frac{\partial}{\partial t} \frac{\rho q_{g}}{\rho_{g}}\right)\right) .
\end{aligned}
$$

This prognostic pressure equation together with the prognostic momentum equation will allow to use an efficient scheme to treat the equations of motion with an implicit scheme (see Section 2.7.2 for details).

\subsection{The turbulence closure}

The choice of the turbulent exchange coefficient is crucial for modelling a volcanic plume. While in an atmosphere at rest these coefficients are of the order of $1 \mathrm{~cm}^{2} / \mathrm{s}$, they can easily grow up to order of $1000 \mathrm{~m}^{2} / \mathrm{s}$ in a fully developed plume. The important aspect of turbulence in and around the plume is that the mixing of air into the particle-laden plume is a crucial mechanism that changes the buoyancy of the plume. This sensitivity has already been discussed by Sparks and Wilson (1976) and Woods (1988) with simplified one-dimensional models. However, there are also extreme cases like pyroclastic flows where the particle-laden plume does not become buoyant quickly through mixing with ambient air despite high temperatures in the interior flow. This is because turbulence is suppressed due to stratification across the plume-air interface and because mixing into a pyroclastic flow is possible only from the top. Such mixing across an interface between fluids of different properties is called entrainment and can be parameterized by a turbulent diffusion coefficient. Following the basic ideas of Kolmogorov (1941) and Prandtl and Wieghardt (1945) the turbulent exchange coefficients are related with the turbulent kinetic energy and the eddy length scale. However, the assumption of an isotropic distribution of turbulent energy cannot hold for the application to a volcanic plume as the production of turbulent energy has no preferred direction of sources and sinks. The idea of Lautenschlager (1983, 1985)

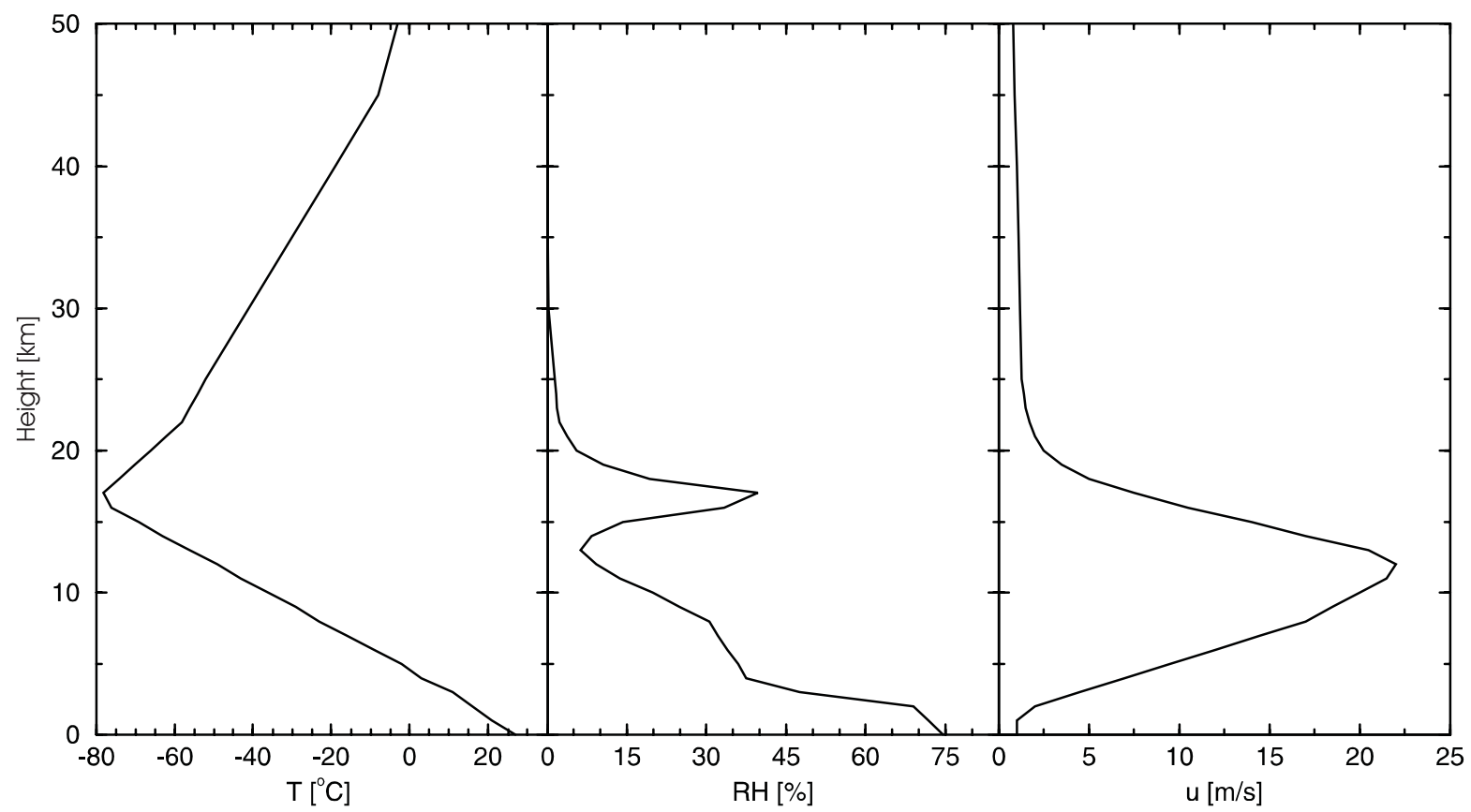

Fig. 1. Meteorological background conditions for the vertical profile of temperature, humidity and horizontal velocity for tropical conditions. 


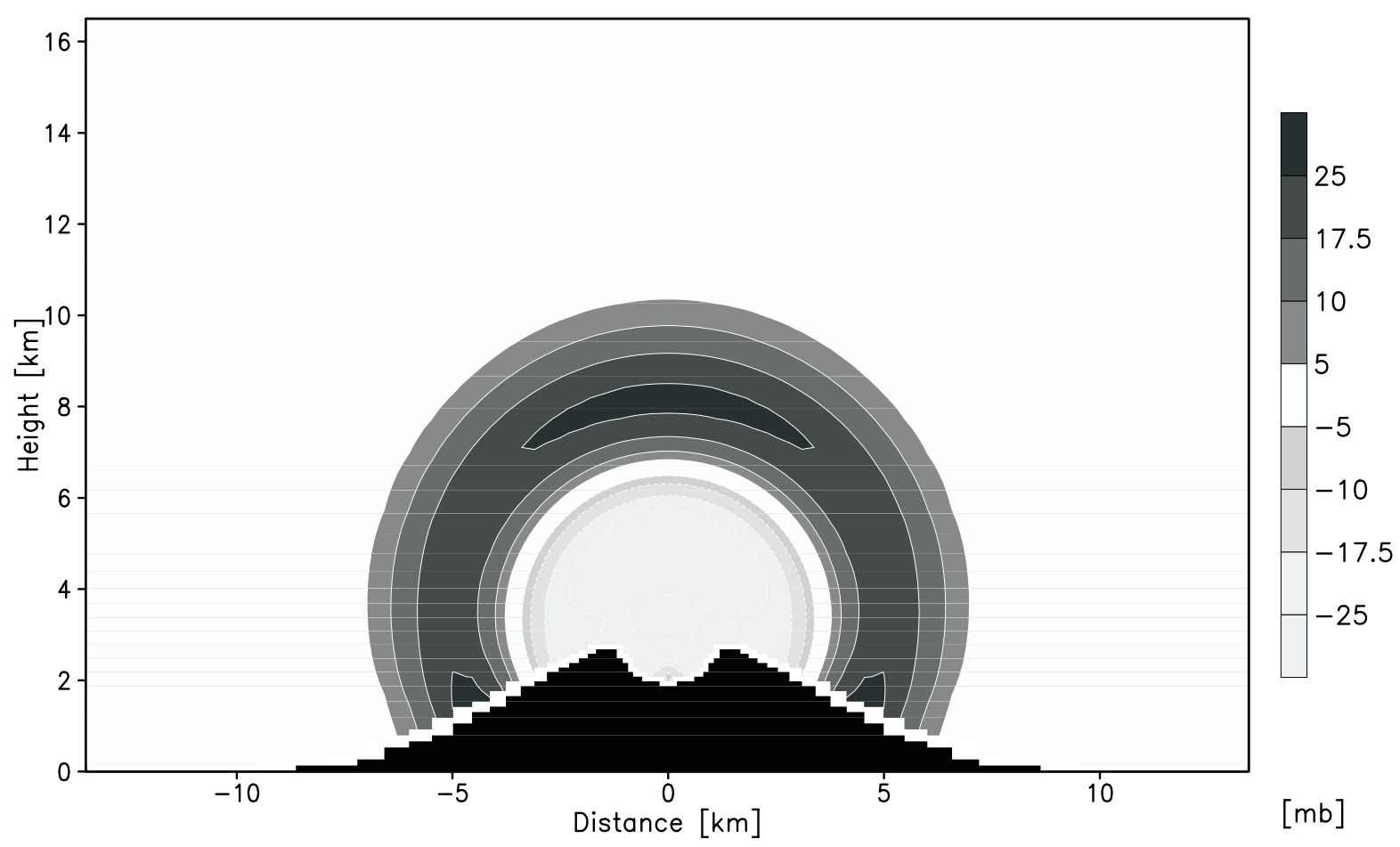

Fig. 2. Pressure anomaly relative to the initial pressure profile for the sound wave propagation experiment $20 \mathrm{~s}$ after the start.

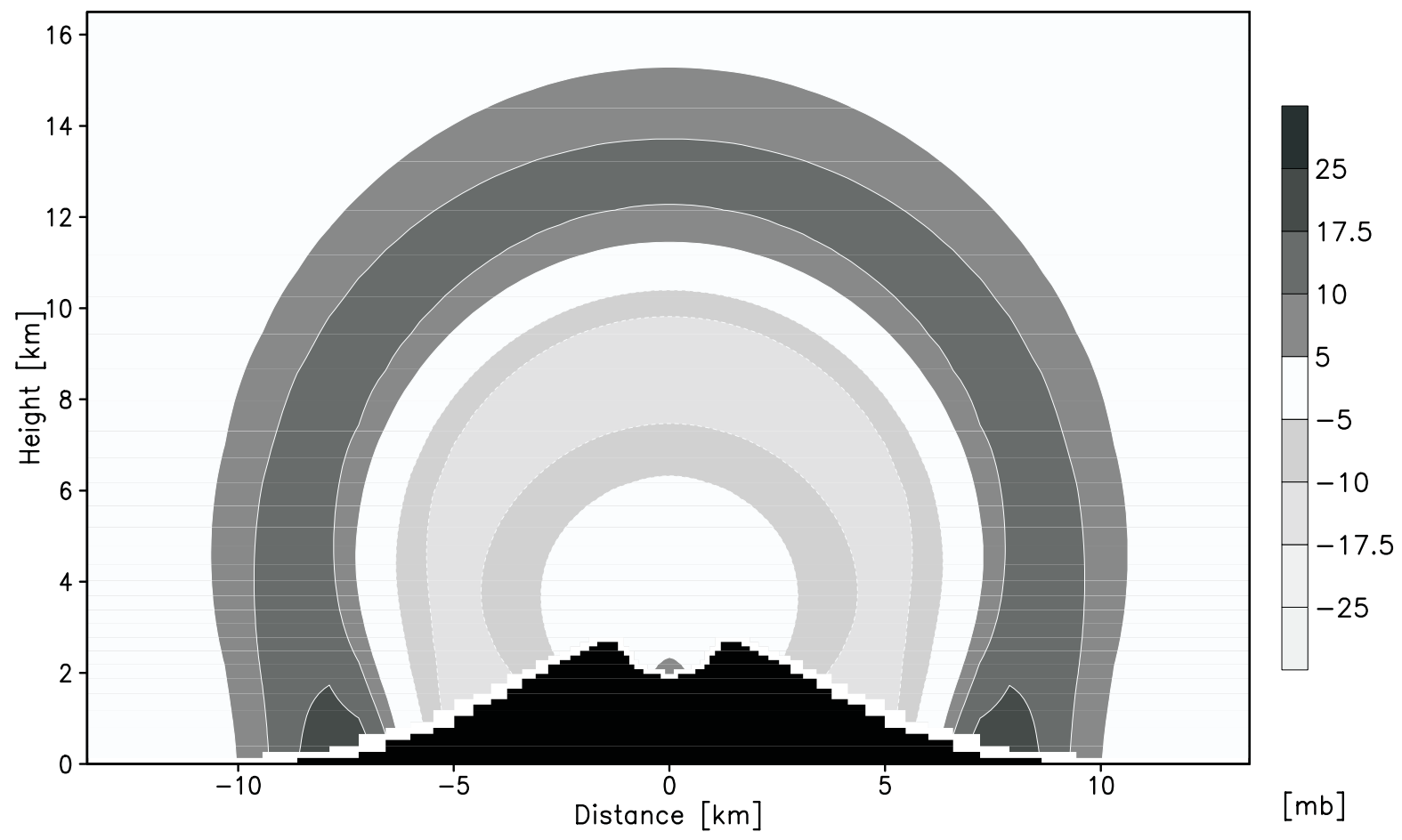

Fig. 3. Pressure anomaly relative to the initial pressure profile for the sound wave propagation experiment $30 \mathrm{~s}$ after the start. 
Table 2

Geometrical and forcing parameters used for the experiments with cylindrical coordinates, where $d_{\mathrm{e}}$ is the diameter of the vent, $F_{\mathrm{e}}$ the size of the domain, $w_{\mathrm{e}}$ the eruption velocity, $T_{\mathrm{e}}$ the eruption temperature, $q_{g, \mathrm{e}}$ the specific gas concentration of the erupted material, $\rho_{\mathrm{e}}$ its volume mean density and $\rho \sum_{n} Q_{n}^{q}$ the total mass flux through the vent

\begin{tabular}{llll}
\hline Experiment & CYL1 & CYL2 & CYL3 \\
\hline$d_{\mathrm{e}}[\mathrm{m}]$ & 60 & 150 & 300 \\
$F_{\mathrm{e}}[\mathrm{km} 2]$ & $7.5 \times 7.5$ & $100 \times 30$ & $200 \times 50$ \\
$w_{\mathrm{e}}[\mathrm{ms}-1]$ & 250 & 205 & 131 \\
$T_{\mathrm{e}}[\mathrm{K}]$ & 1200 & 1011 & 727 \\
$q_{g, \mathrm{e}}[\mathrm{kg} / \mathrm{kg}]$ & 0.03 & 0.205 & 0.491 \\
$\rho_{\mathrm{e}}\left[\mathrm{kg} \mathrm{m}{ }^{-3}\right]$ & 5.52 & 1.31 & 0.80 \\
$\rho \sum_{n} Q_{n}^{q}\left[10^{6} \mathrm{~kg} \mathrm{~s}^{-1}\right]$ & 3.90 & 4.75 & 7.42 \\
\hline
\end{tabular}

to derive a set of prognostic equations for the horizontal and vertical component of the turbulent kinetic energy has finally been extended to also predict rather than diagnose the turbulent length scale.

\subsubsection{Prognostic equations}

The prognostic equations for the horizontal and vertical components of the turbulent kinetic energy
$B_{\text {hor }}$ and $B_{\text {ver }}$, respectively, are:

$$
\begin{aligned}
\frac{\partial}{\partial t} B_{\mathrm{hor}}= & -u_{i} \frac{\partial}{\partial x_{i}} B_{\mathrm{hor}}+\frac{1}{\rho} \frac{\partial}{\partial x_{i}} \rho c_{1} K_{i}^{m} \frac{\partial}{\partial x_{i}} B_{\mathrm{hor}} \\
& +2 K_{i}^{m}\left(\frac{\partial}{\partial x_{i}} u_{1}\right)^{2}+2 K_{i}^{m}\left(\frac{\partial}{\partial x_{i}} u_{2}\right)^{2} \\
& -c_{2} \frac{\sqrt{B}}{L}\left(B_{\mathrm{hor}}-\frac{2}{3} B\right)-c_{3} \frac{\sqrt{B}}{2 L} B_{\mathrm{hor}} \\
\frac{\partial}{\partial t} B_{\mathrm{ver}}= & -u_{i} \frac{\partial}{\partial x_{i}} B_{\mathrm{ver}}+\frac{1}{\rho} \frac{\partial}{\partial x_{i}} \rho c_{1} K_{i}^{m} \frac{\partial}{\partial x_{i}} B_{\mathrm{ver}} \\
& +2 K_{i}^{m}\left(\frac{\partial}{\partial x_{i}} u_{3}\right)^{2}-2 g K^{\sigma} \frac{1}{\sigma} \frac{\partial}{\partial z} \sigma \\
& -c_{2} \frac{\sqrt{B}}{L}\left(B_{\mathrm{ver}}-\frac{1}{3} B\right)-c_{3} \frac{\sqrt{B}}{L} B_{\mathrm{ver}}
\end{aligned}
$$

where $L$ is the turbulence length scale, $B=B_{\text {hor }}+$ $B_{\text {ver }}$ is the total turbulent kinetic energy, and $c_{1}=0.8$

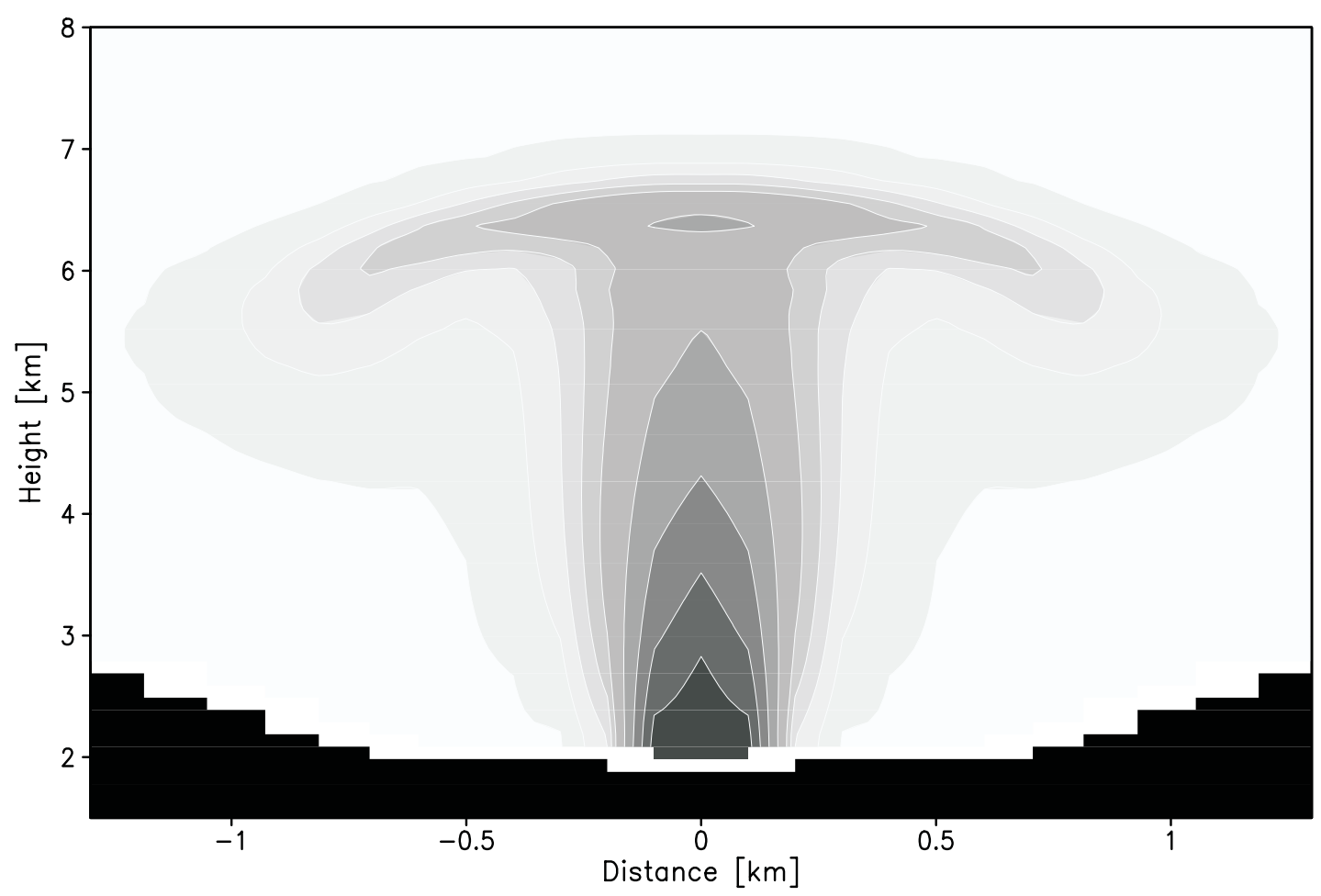

Fig. 4. Specific concentration of ash for the experiment CYL3 with cylindrical coordinates after $110 \mathrm{~s}$. 


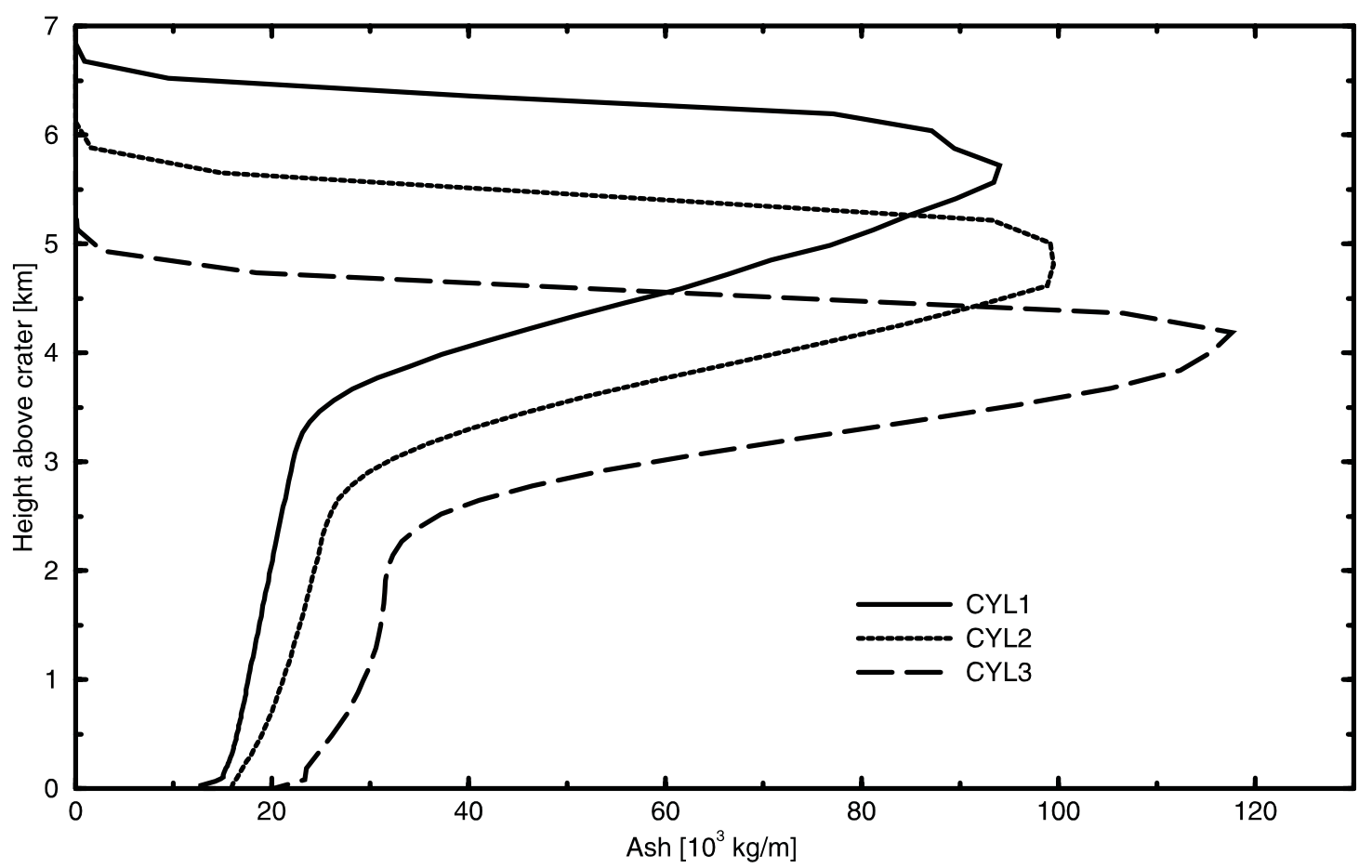

Fig. 5. Vertical profile of horizontally integrated ash concentration for the experiments CYL1, CYL2 and CYL3 after $110 \mathrm{~s}$.

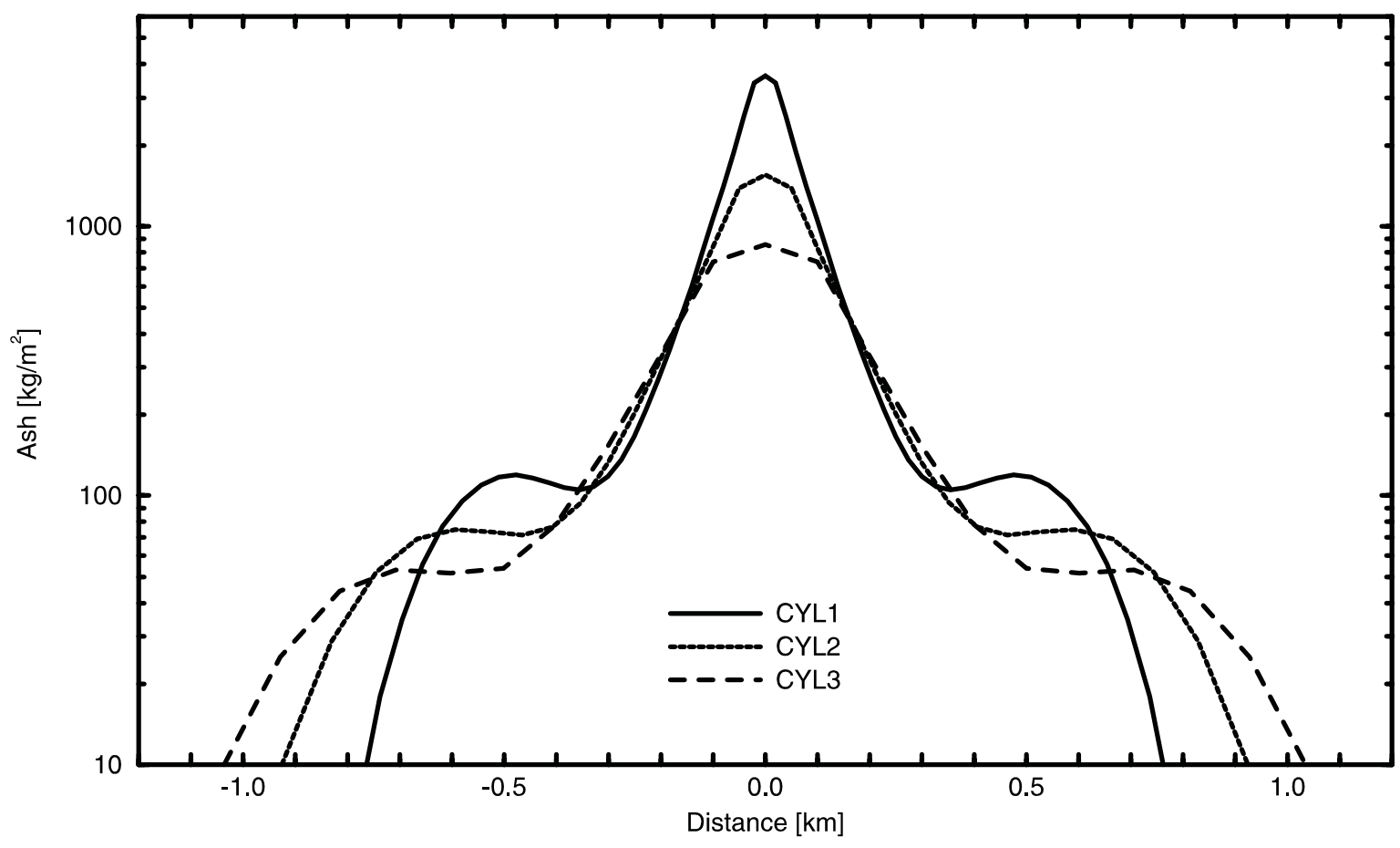

Fig. 6. Horizontal profile of vertically integrated ash concentration for the experiments CYL1, CYL2 and CYL3 after $110 \mathrm{~s}$. 
and $c_{2}=0.43$ are empirical constants according to Zilitinkevich et al. (1967), Mellor and Yamada (1974), Yu (1976) and Lewellen (1977). $c_{3}$ is defined in Section 2.4.2. The static stability is defined using the potential density $\sigma$, which is the density of the fluid brought to a common pressure level:

$$
\frac{1}{\sigma}=q_{g} \frac{R_{g}}{p_{0}} \Theta_{g}+\sum_{n}^{n \neq g} \frac{q_{n}}{\rho_{n}}
$$

The turbulent length scale is considered to be a conservative quantity in the absence of sources and sinks (Daly and Harlow, 1970). A prognostic advection-diffusion equation in advective form is used to consider regimes where typical time scales of generation and dissipation of turbulence are longer than the time scale for the flow to cross a grid cell. The equation is:

$$
\begin{aligned}
\frac{\partial}{\partial t} L= & -u_{i} \frac{\partial}{\partial x_{i}} L+\frac{1}{\rho} \frac{\partial}{\partial x_{i}} \rho c_{1} K_{i}^{m} \frac{\partial}{\partial x_{i}} L \\
& -c_{2} \frac{\sqrt{B}}{L}\left(L-L_{0}\right)
\end{aligned}
$$

where $L_{0}$ is the turbulent equilibrium length scale, which according to the approach by Deardorff (1980) is defined as:

$L_{0}=0.54 \sqrt{\frac{B}{N^{2}}}$ with $N^{2}=-\frac{g}{\sigma} \frac{\partial}{\partial x_{3}} \sigma$

where $N^{2}$ is the Brunt-Vaisalla frequency. $L_{0}$ is set to $\Delta s=(\Delta x+\Delta y+\Delta z) / 3$ for unstable conditions, i.e., $N^{2}<0$, or if $L_{0}$ exceeds $\Delta s$. The latter threshold is needed in order not to put turbulent energy into scales that are well resolved by the grid. $\Delta x$, $\Delta y$ and $\Delta z$ are the grid distances in the three dimensions.

\subsubsection{Diagnostic equations}

Typical flows in a volcanic plume approach or even exceed speed of sound, thus effects of compressibility need to be considered. Following Deardorff (1980) and including a dependence on the

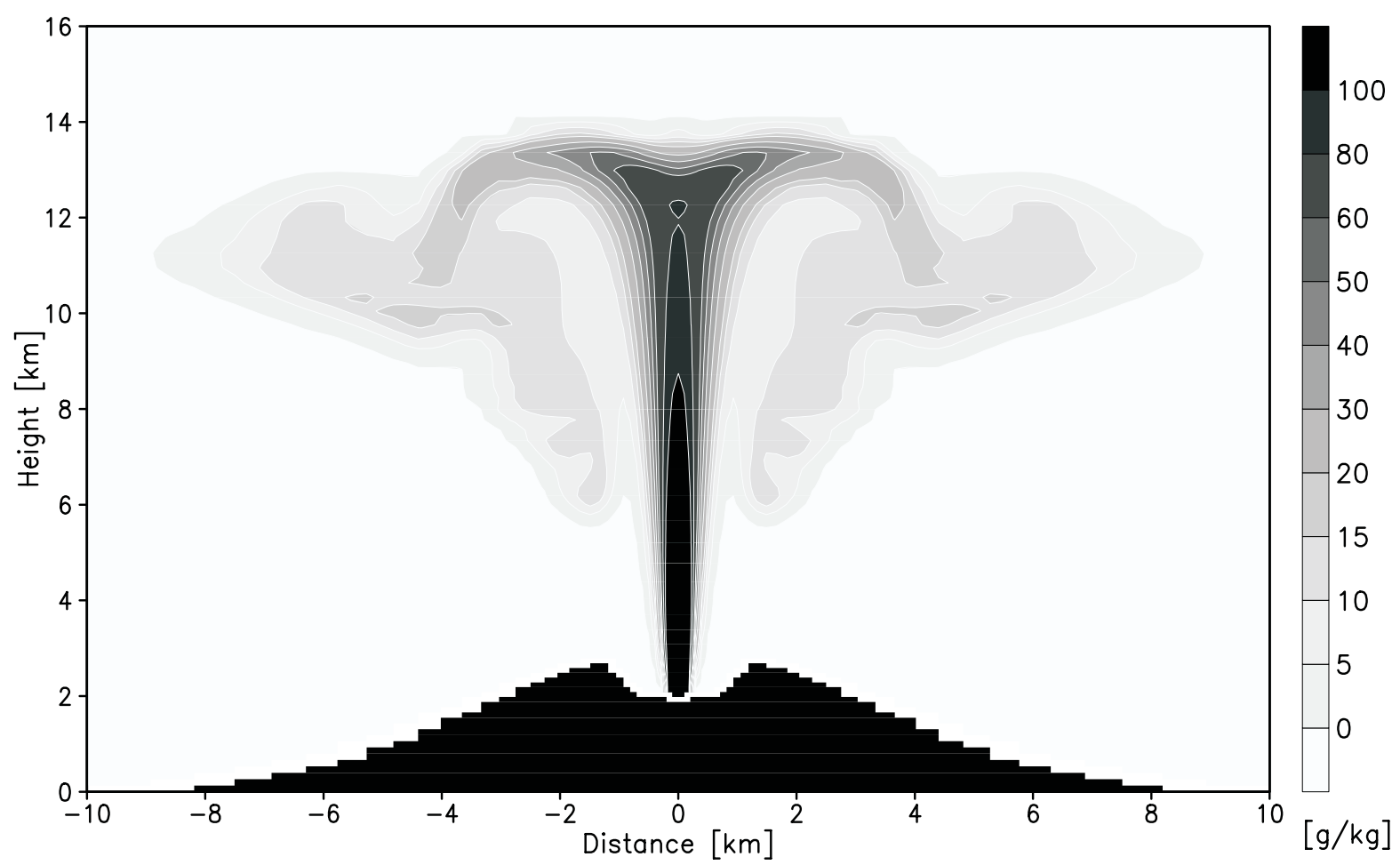

Fig. 7. Specific concentration of ash for the experiment CYL3 with cylindrical coordinates after 25 min. 
turbulent Mach-number $M_{t}$ according to Zeman (1990, 1992), $c_{3}$ is defined as:

$$
\begin{aligned}
c_{3}= & \left(0.067+0.18 \frac{L}{\Delta s}\right)\left(1+\frac{3}{4}(1\right. \\
& -\exp \left(-\left(\frac{M_{t}-0.1}{0.6}\right)^{2}\right) \text { with } M_{t}=\frac{B}{c_{s}}
\end{aligned}
$$

where $c_{3}$ now contains the correction for the dissipation and redistribution of turbulent energy (see Eqs. (22) and (23)) by the turbulent Mach-number $M_{t}$. The speed of sound $c_{s}$ is defined by the wave equation which results when the flux divergence in Eq. (21) is replaced by the left-hand side of the momentum equation (Eq. (3)). It is found that in this model $c_{s}$ is:

$c_{s}=\sqrt{\frac{c_{p, g}}{c_{v, g}}\left(c_{p, g}-c_{v, g}\right) T \frac{V}{V_{g}} q_{g}}$

This equation compares well with the approximation by Woods (1995).

Because particle properties are quite variable and big corrections to the Prandtl-numbers with respect to static stability, for example, are needed and quite uncertain, no further correction for them have been included. The Prandtl-numbers for heat $\alpha_{\Theta}$, for tracers $\alpha_{q}$ and for turbulence $\alpha_{\sigma}$ are:

$$
\left(\alpha_{\Theta}, \alpha_{q}, \alpha_{\sigma}\right)=\left(1+2 \frac{L}{\Delta s}, \frac{3}{4}, \alpha_{\Theta} q_{g}+\sum_{n}^{n \neq g} \alpha_{q} q_{n}\right)
$$

Finally, the turbulent exchange coefficients for momentum $K_{i}^{m}$, for heat $K_{i}^{\Theta}$, for tracers $K_{i}^{q}$ and for turbulence $K_{\sigma}$ can be determined from:

$$
\begin{aligned}
& \left(K_{1}^{m}, K_{2}^{m}, K_{3}^{m}\right) \\
& =\left(c_{0} L \sqrt{\frac{3}{2} B_{\mathrm{hor}}}, c_{0} L \sqrt{\frac{3}{2} B_{\mathrm{hor}}}, c_{0} L \sqrt{3 B_{\mathrm{ver}}}\right) \\
& \left(K_{1}^{\Theta}, K_{2}^{\Theta}, K_{3}^{\Theta}\right)=\left(\alpha_{\Theta} K_{1}^{m}, \alpha_{\Theta} K_{2}^{m}, \alpha_{\Theta} K_{3}^{m}\right) \\
& \left(K_{1}^{q}, K_{2}^{q}, K_{3}^{q}\right)=\left(\alpha_{q} K_{1}^{m}, \alpha_{q} K_{2}^{m}, \alpha_{q} K_{3}^{m}\right) \\
& K^{\sigma}=\alpha_{\sigma} K_{3}^{m}
\end{aligned}
$$

where $c_{0}=0.32$ is a further empirical constant.

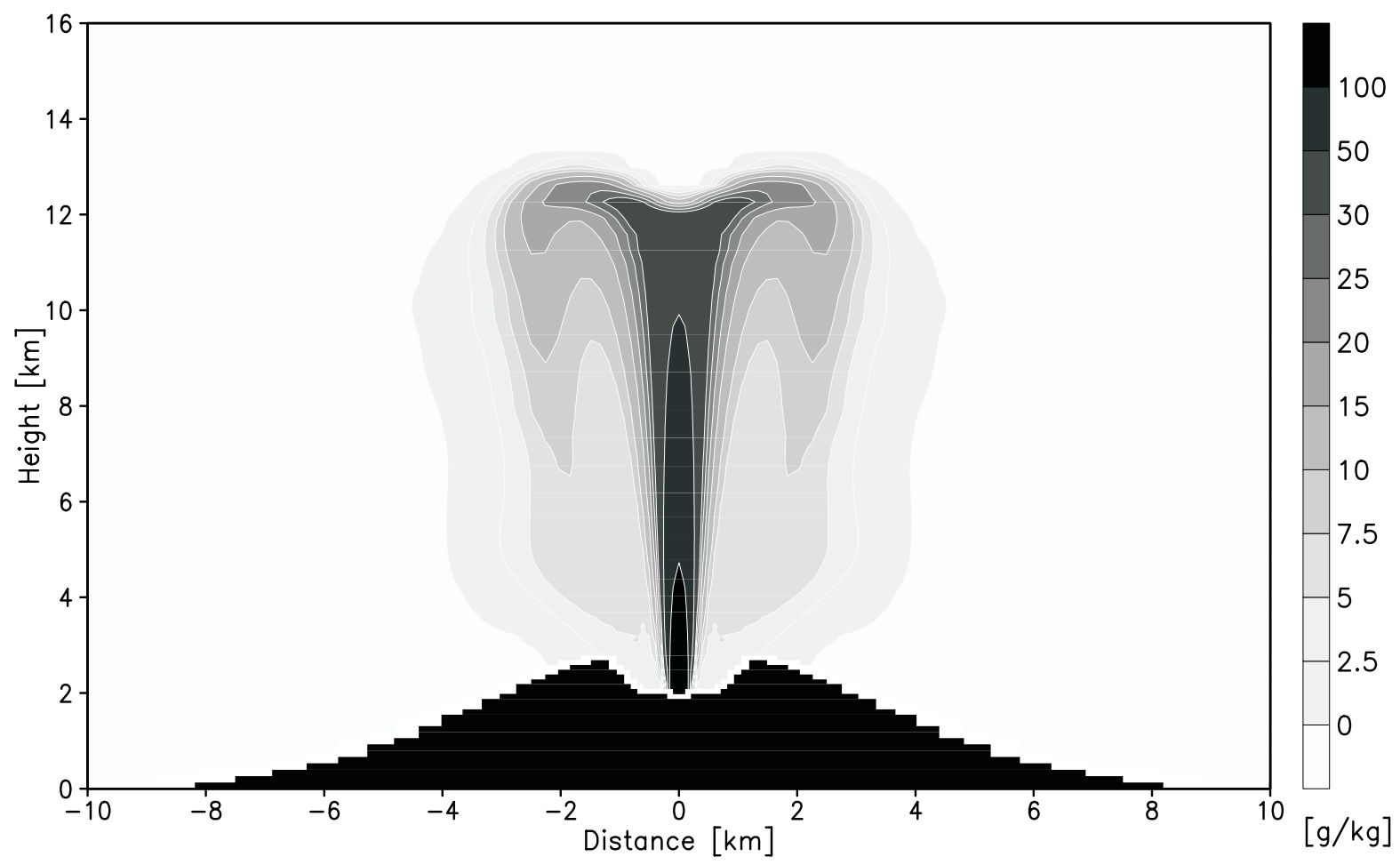

Fig. 8. Specific concentration of lapilli for the experiment CYL3 with cylindrical coordinates after 25 min. 


\subsection{Microphysics}

The microphysical package is one of the important components within this model concept, which allows for the simulation of the development and propagation of a plume vertically up to high altitudes and horizontally over large distances. This is because in longer integrations water vapor condenses to cloud water or sublimates to ice crystals. The processes may produce rain, i.e., precipitable water, or form graupel. These are crucial processes when chemical reactions and dissolution processes need to be modeled. The latent heat and mass transfer between the modeled components serve as forcing for the dynamics and thermodynamics. The forcing of the total heat content $Q^{\Theta}$ and of the tracer masses $Q^{n}$, and the relative fallout velocity $\Delta u_{3, n}$ complete the set of thermo-hydrodynamic equations and, for convenience, are fully described and discussed in a companion paper by Herzog et al. (1998).

\subsection{Large-scale atmospheric background state}

As the model's concept is to perform integrations over more than an hour this is an opportunity to also study the link between the large-scale flow and other conditions like the profile of temperature and humidity far up into the stratosphere. Confirmed by a recent study of Glaze and Baloga (1996) the largescale background state of the atmosphere, i.e., the current weather regime, has significant implications on the development of a volcanic plume. Various representative atmospheric profiles have been determined from the ECMWF re-analysis project (Gibson et al., 1997). The wind profile is obtained from these data. The temperature and humidity profiles are taken from McClatchey et al. (1972). An example for a tropical profile is shown in Fig. 1. These profiles are used to initialize the atmospheric state for temperature, pressure and humidity, and are used as lateral and top boundary condition throughout an experi-

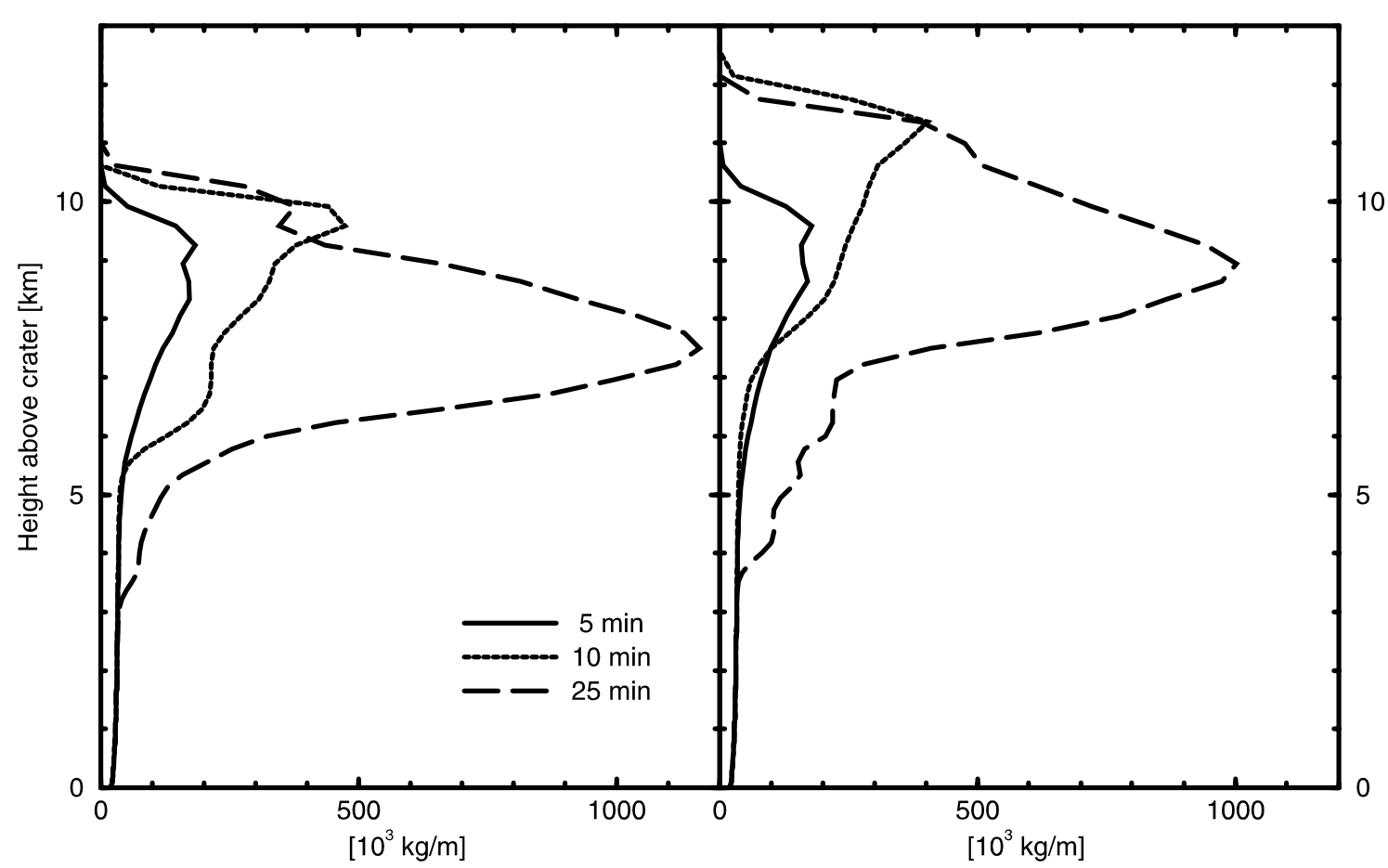

Fig. 9. Vertical profile of horizontally integrated ash concentration for the experiment CYL3 after 5, 10 and 25 min after the start of the eruption. The left-hand side frame is for the case without microphysics, the right-hand side includes it. 
ment. The background wind profile $u_{k}^{*}$ is applied in the entire model domain via the Coriolis force term in Eq. (3). Because these single profiles do not consider any chosen orography, the model has to be brought to equilibrium through a spinup run without any volcanic forcing. This guarantees a realistic boundary layer which is then used as the initial state to perform all the experiments.

\subsection{Numerical solution}

\subsubsection{The grid}

The finite-differencing equations are formulated for a regular Arakawa C-grid (Mesinger and Arakawa,1976), which is suitable for compressible flow. Due to the need to cover hundreds of kilometers horizontally and nearly a hundred kilometers vertically, a variable grid spacing was introduced that varies from typically $100 \mathrm{~m}$ or less near the vent to several kilometers near the lateral boundary and 1 $\mathrm{km}$ below the top boundary. The transition between low and high resolution is carried out by minimizing the change of the grid size and thus by minimizing numerical instabilities that might occur when the grid distance is variable.

\subsubsection{The time step scheme}

The basic concept used in this model for implicitly solving the wave equation in terms of a prognostic pressure equation is that of Kwizak and Robert (1971). However, we use a two-time level rather than a three-time level scheme for efficiency reasons. The underlying idea to derive a prognostic equation for the pressure is presented in Appendix A

If the momentum equation (Eq. (3)) is used to eliminate the divergence operator in Eq. (21) then a prognostic equation for the gas pressure is obtained. This is possible as $u_{i}$ and $u_{i, g}$ differ only by the diagnostically determined vertical slip velocity. Also the approximation of dynamic equilibrium (Eq. (1)) assumes that the volume mean pressure $p$ is identical to the gas pressure $p_{g}$.

In the context of the pressure and tracer equation the problem is that the volume mean density $\rho$ needs

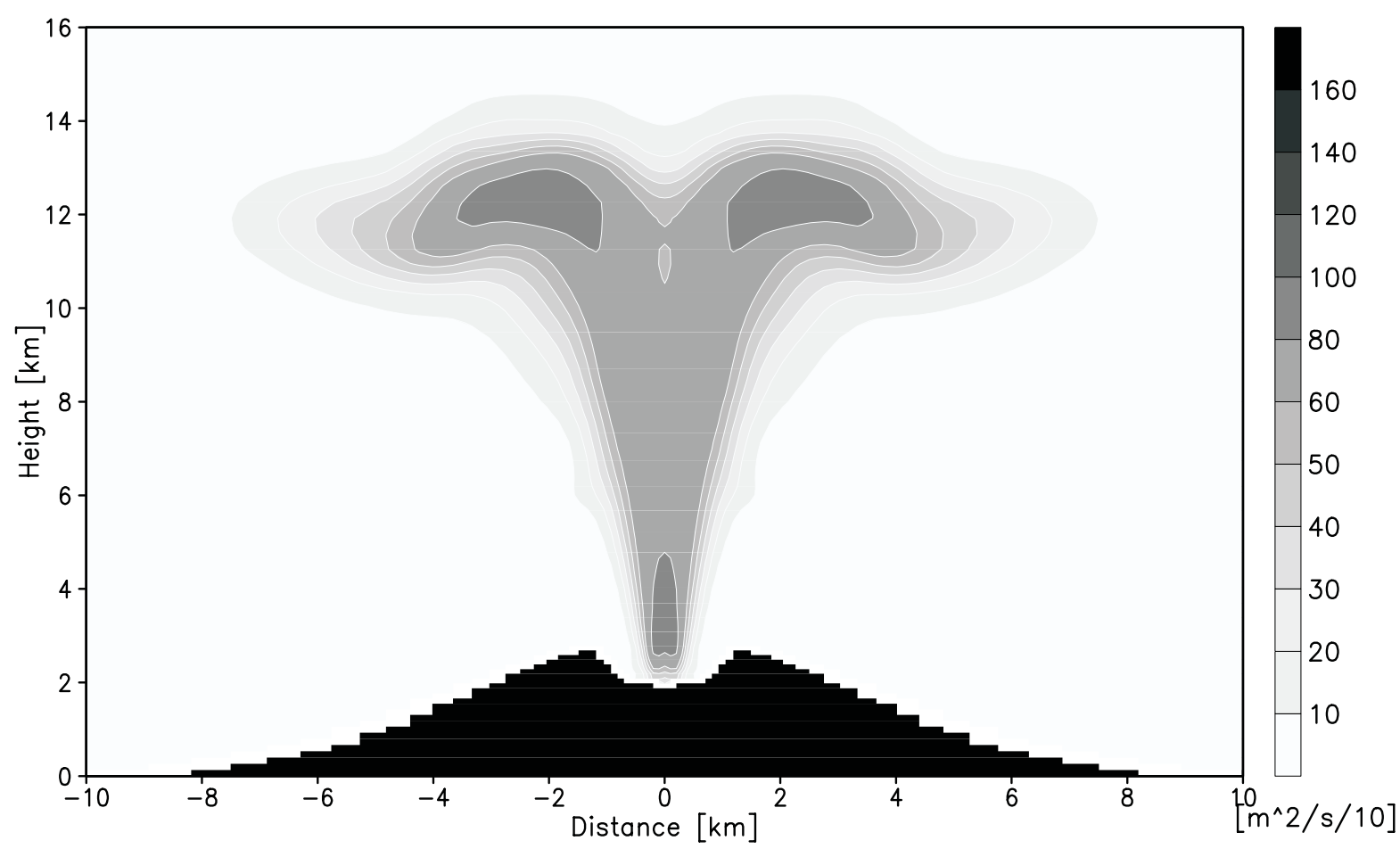

Fig. 10. Horizontal turbulent exchange coefficient for the experiment CYL3 with cylindrical coordinates after 25 min. 
to be determined before all other quantities can be computed, otherwise mass conservation cannot be guaranteed. On the other hand the specific concentrations $q_{n}$ at the new time level need to be available before $\rho$ can be computed. This conflict is solved by a parallel iteration of all quantities. In detail, the solution consists of four steps. In the first step, a single iteration for each of the tracers $q_{n}$ and total heat $\Theta$ is carried out. In the second step, densities for gas and the volume mean are determined diagnostically. In the third step, a single iteration for the wave equation is performed in order to determine a guess for the flow field $u_{i}$ and pressure field $p$. In the last step, an implicit correction step for momentum guarantees that the solution is also stable for advection and diffusion. This four-step procedure is repeated until convergence is achieved. It was found that it converges within less than 10 iterations.

A key algorithm that helps find the solutions quickly is the alternating-direction-line-successiveoverrelaxation ADLSOR scheme. It is used to solve the elliptic equations for wave propagation, advec- tion and diffusion of momentum, tracers and heat. This scheme has been found to be very efficient in previous models (O’Brien, 1986; Luther, 1986). For a two-dimensional elliptic equation it can be understood as a one-dimensional iteration, where the solution is a vector obtained from a system of linear equations, which is usually based on a tridiagonal matrix resulting from second order central differencing. The latter is called line-relaxation. Alternatingdirection means that the direction of direct solution is interchanged for each iteration, thus a satisfying convergence to the solution is achieved in both directions. Finally, successive overrelaxation denotes the standard method to speed-up convergence (see also Oberhuber, 1993).

\subsubsection{The transport scheme}

The transport of tracers is carried out with a scheme similar to that developed by Smolarkiewicz (1984). However, centred differencing is chosen as initial step rather than an upwind scheme. The diffusive step has the task to guarantee positive values.

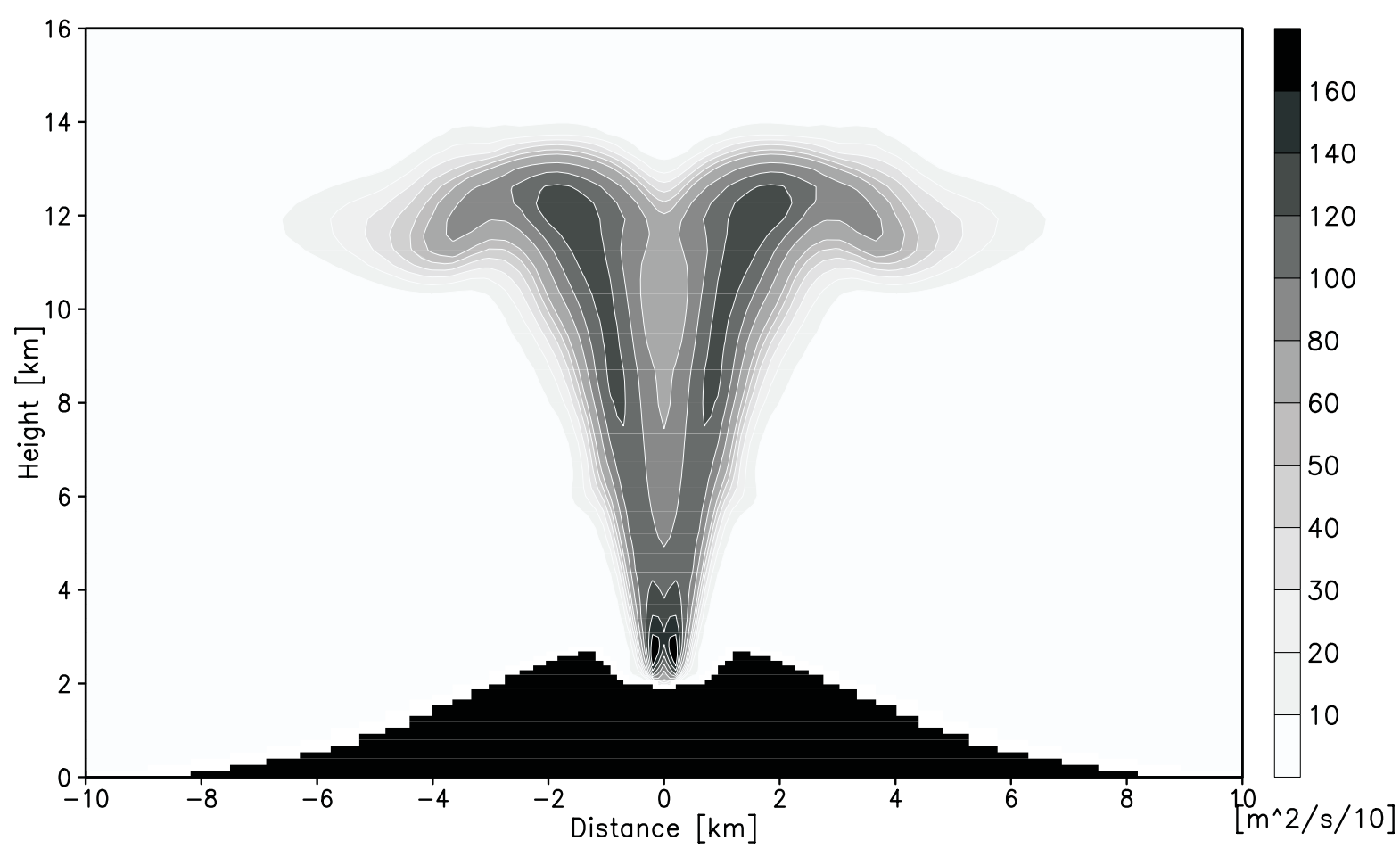

Fig. 11. Vertical turbulent exchange coefficient for the experiment CYL3 with cylindrical coordinates after 25 min. 
The scheme is mass conserving, positive definite and has little implicit diffusion. The advection of heat is carried out by a scheme very similar to Crowley (1968) and is discussed by Smolarkiewicz (1985).

\subsubsection{Boundary conditions}

Through the use of flags as indicated in Eq. (36) an arbitrary orography can be introduced. At the earth's surface a no-slip boundary condition is employed. The input through the vent is defined as an additional vertical velocity that transports dry gas, water vapor, ash and lapilli with a specified concentration across the bottom of the vent. In addition, a horizontal and vertical component of turbulent energy, as well as the turbulence's length scale are specified.

The lateral boundary conditions are determined from the atmospheric background profile for flow, temperature and water vapor in the case of inflow. For outflow conditions, the predicted model quantities are advected towards the boundaries. The pressure profile is calculated from temperature and water vapor profile using the hydrostatic approximation. The upper boundary is set to a constant pressure. The flow next to the boundaries is computed using the full equations for momentum. This is possible due to the staggering of the grid. A simple nudging towards the prescribed background profile just below the upper boundary absorbs waves that are excited, e.g., during the start of the eruption. A further discussion of the accuracy of the pressure boundary conditions can be found in Appendix B.

\subsubsection{Test for sound wave propagation}

A simple test to evaluate the numerical treatment of compressible flow is the generation of a sound wave in the crater. In an experiment, the instantaneous injection of dry air into the crater leads to an explosion and a subsequent propagation of a sound wave in the atmosphere. Figs. 2 and 3 show the pressure anomaly relative to that of the resting air 20 and $30 \mathrm{~s}$ after the explosion, respectively. An inspection of the numbers shows that the sound wave decays linearly with distance. This is because in this

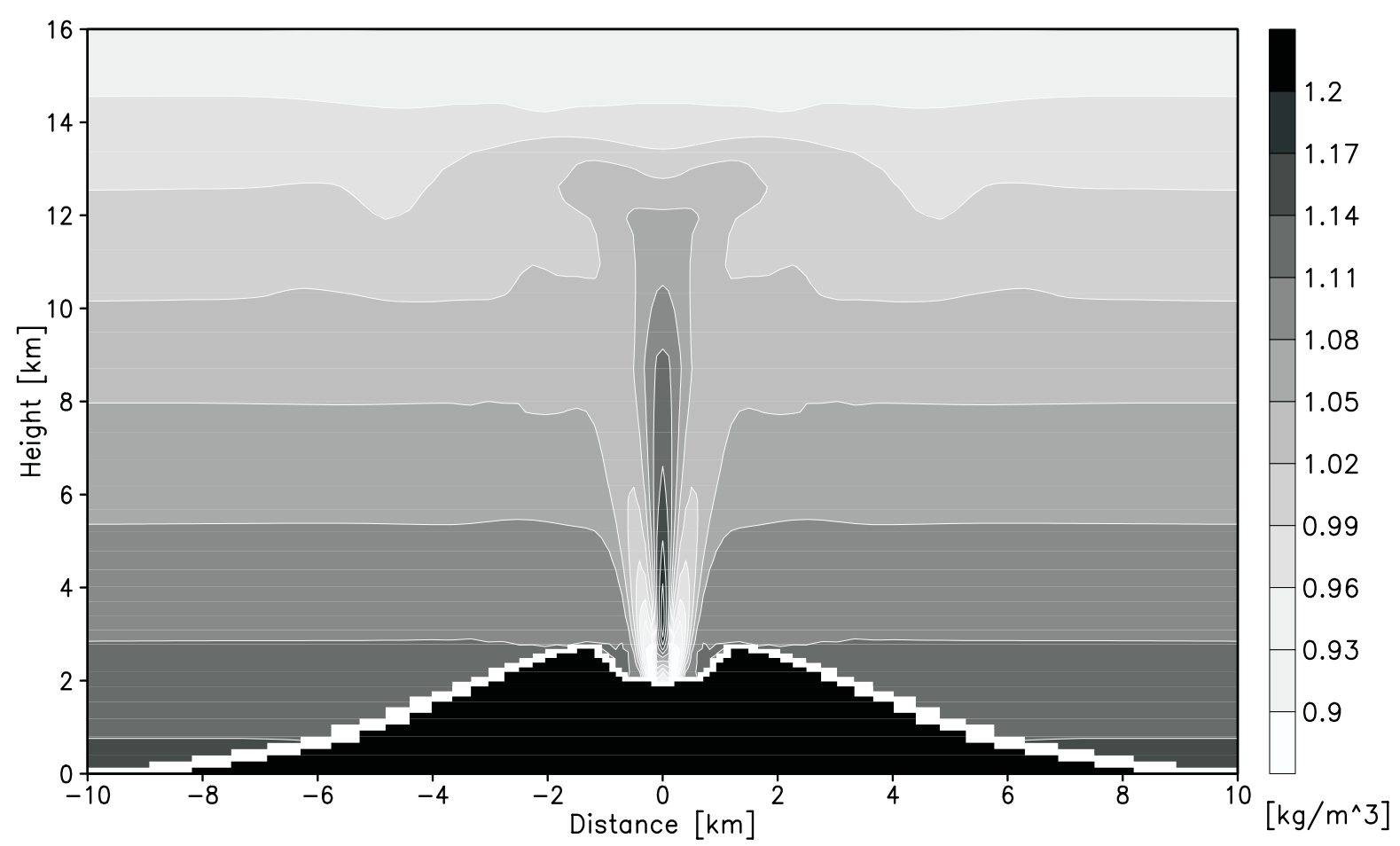

Fig. 12. Potential density for the experiment CYL3 with cylindrical coordinates after 25 min. 
two-dimensional experiment the eruption is considered to be homogenous in the third unresolved direction. The result is qualitatively comparable with Dobran et al. (1993) in that the reflection of upward moving energy due to the decreasing speed of sound with altitude creates a maximum in the pressure anomaly above the ground.

\section{Model experiments}

Despite the model being formulated in three-dimensions this paper concentrates on various two-dimensional experiments. The questions to be addressed are the dependence of the solution on grid resolution, geometry and atmospheric background conditions. Emphasis is also put on the role of microphysics and of turbulence. In all subsequent simulations the ash's particle density, radius and slip velocity at $1000 \mathrm{~h} \mathrm{~Pa}$ are $1500 \mathrm{~kg} \mathrm{~m}^{-3}, 0.06 \mathrm{~mm}$ and $0.64 \mathrm{~ms}^{-1}$, respectively. For lapilli, at the same pressure level the particle density is $1000 \mathrm{~kg} \mathrm{~m}^{-3}$, the radius $2.00 \mathrm{~mm}$ and the slip velocity $7.62 \mathrm{~ms}^{-1}$. Lapilli hold a third of the total solid mass. All microphysical quantities are defined in detail in the work of Herzog et al. (1998).

\subsection{The grid layout}

The model area covers $200 \mathrm{~km}$ horizontally and $50 \mathrm{~km}$ vertically, unless otherwise stated. The horizontal extent is necessary in simulations where the mean wind is carrying the plume towards the boundary within a typical simulation time of an hour. Initial sensitivity tests for simulating the same solution with a varying top boundary altitude yield the conclusion that the top boundary has to be chosen far above the highest vertical extent of the plume. If this is not done, there is not enough fluid above the plume that can respond with sinking motion and thus with cooling above the plume in order to act as a brake on the rising plume. The horizontal grid resolution varies from $100 \mathrm{~m}$ near the vent to $5 \mathrm{~km}$ at the boundary, the vertical resolution varies from $100 \mathrm{~m}$

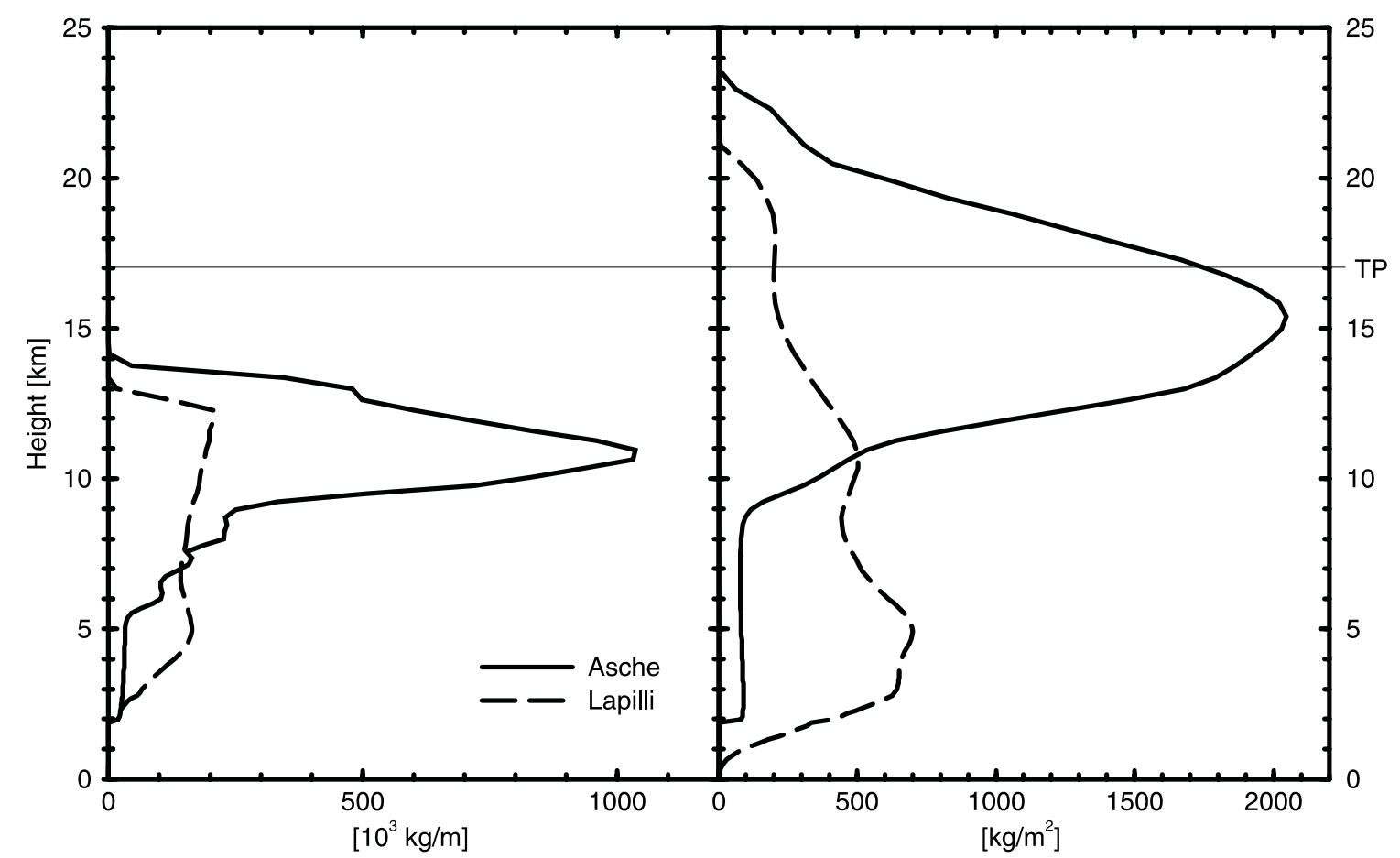

Fig. 13. Vertical profile of horizontally integrated ash and lapilli concentration for the experiment CYL3 with cylindrical coordinates left-hand side and for a line-volcano right-hand side after $25 \mathrm{~min}$. Note that the abscissa dimensions are different for the two cases. 
at the surface to $1 \mathrm{~km}$ at the top boundary. This setup has $127 \times 127$ grid points and is used for all experiments unless otherwise mentioned. The crater is represented by a $3 \mathrm{~km}$ high symmetric mountain and a caldera, so that the vent is located at an altitude of $2 \mathrm{~km}$.

The time step $\Delta t$ is variable. It is computed for each time step such that the ratio between maximum flow speed and grid distance divided by the model's time step does not exceed 0.8 . Note that due to the implicit time-stepping for the wave equation there is no stability limit for waves. This means that during the spin-up with a background wind but without eruption, $\Delta t$ is a few seconds, while during an eruption it might be some tenths of a second.

\subsection{Idealized eruption}

In this section, symmetric eruptions, i.e., without background wind, are discussed in regard to the influence of grid resolution and the importance of the three-dimensionality for the plume development.
Three sets of experiments have been performed with varying grid resolutions. The aim is to adjust a realistic forcing with its typical small spatial scales to a coarser resolution model grid, and at the same time to obtain results comparable with those of a fine-resolution model. According to Woods and Bower (1995) the jet leaves the crater at 1.8 times the speed of sound. In the case of a Plinian eruption and assuming a temperature of $T=1200 \mathrm{~K}$, a specific gas concentration of $q_{g}=0.03$ (Woods, 1995) and using Eq. (28) for the speed of sound, the mixture exits the crater at about $250 \mathrm{~ms}^{-1}$. In order to make high resolution runs with a narrow vent experiment CYL1 comparable with coarser resolution experiments with an unrealistic wide vent, a pre-eruptive entrainment of surrounding air is performed. This entrainment is carried out as an off-line calculation. Assuming a horizontal extent of the mature plume equal to the diameter of the vent in the fine-resolution case, as much surrounding air is entrained into the plume as needed to expand the plume to the diameter in the coarse-resolution case.

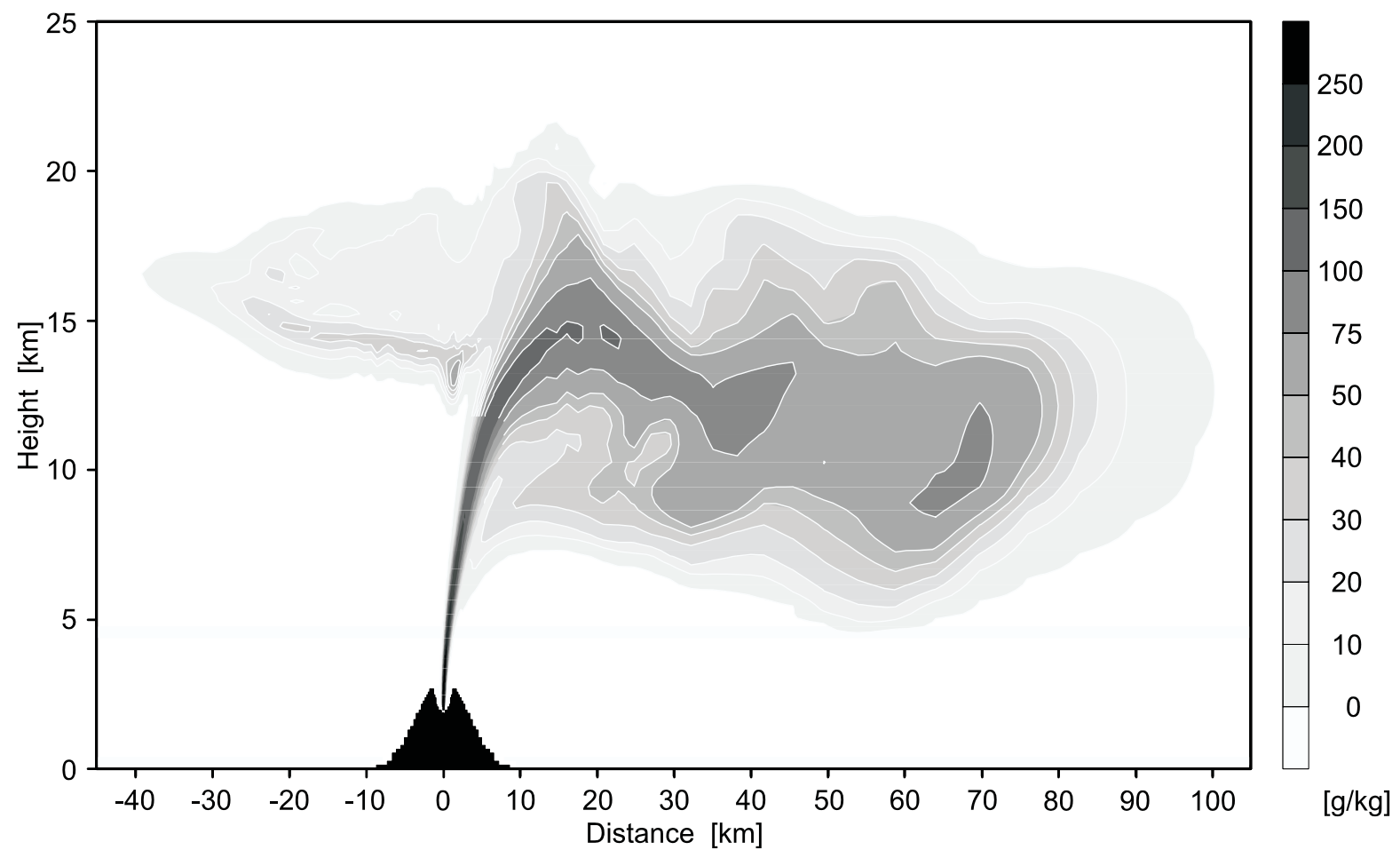

Fig. 14. Specific concentration of ash for an experiment with tropical atmospheric background conditions after 25 min. 
The properties of the entrained air are taken from the observed profiles. Assuming that the fluxes of the solid material's mass are equal and energy and momentum are comparable, the properties of the mixture summarized in Table 2 have been calculated. These are used as lower boundary conditions for the model grids used in CYL3, which is the coarse resolution case and for an increasing resolution near the vent in CYL2. For the higher resolution cases a smaller model domain is used. For the coarse resolution case CYL3, Fig. 4 shows the development of a plume with spreading at the top, which is qualitatively comparable to earlier studies by Valentine and Wohletz (1989). However, Fig. 5 highlights the strong dependence of the plume height on the model's grid resolution. According to Table 2 the high resolution experiment CYL1 uses a much higher eruption velocity, temperature and density when compared with CYL2 or CYL3. Fig. 6 shows the horizontal distribution of the vertically integrated mass of ash. Since the high resolution case CYL1 creates the highest plume the mass is concentrated over the vent while in the lower resolution cases the plume starts to spread out horizontally at an earlier stage of the plume development. The cause is the much bigger initial circumference of the plume for the low resolution case and a much more rapid decay of the density, temperature and upward velocity due to entrainment.

Due to the limited size of the domain for the runs CYL1 and CYL2, only CYL3 has been run for longer time periods. Fig. 7 shows the ash distribution after $25 \mathrm{~min}$. The top of the plume has reached an altitude of $13 \mathrm{~km}$ above the vent with sedimentation of ash on both sides of the upwind area. The lapilli concentration is shown in Fig. 8. In comparison with the sedimentation of the ash, bigger particles immediately settle before they can spread out horizontally with the diverging flow at the top of the plume.

In order to demonstrate the differences in the plume simulation with and without microphysics, Fig. 9 compares the vertical profiles of horizontally integrated ash mass. While the profiles are nearly identical $5 \mathrm{~min}$ after the start of the eruption, the

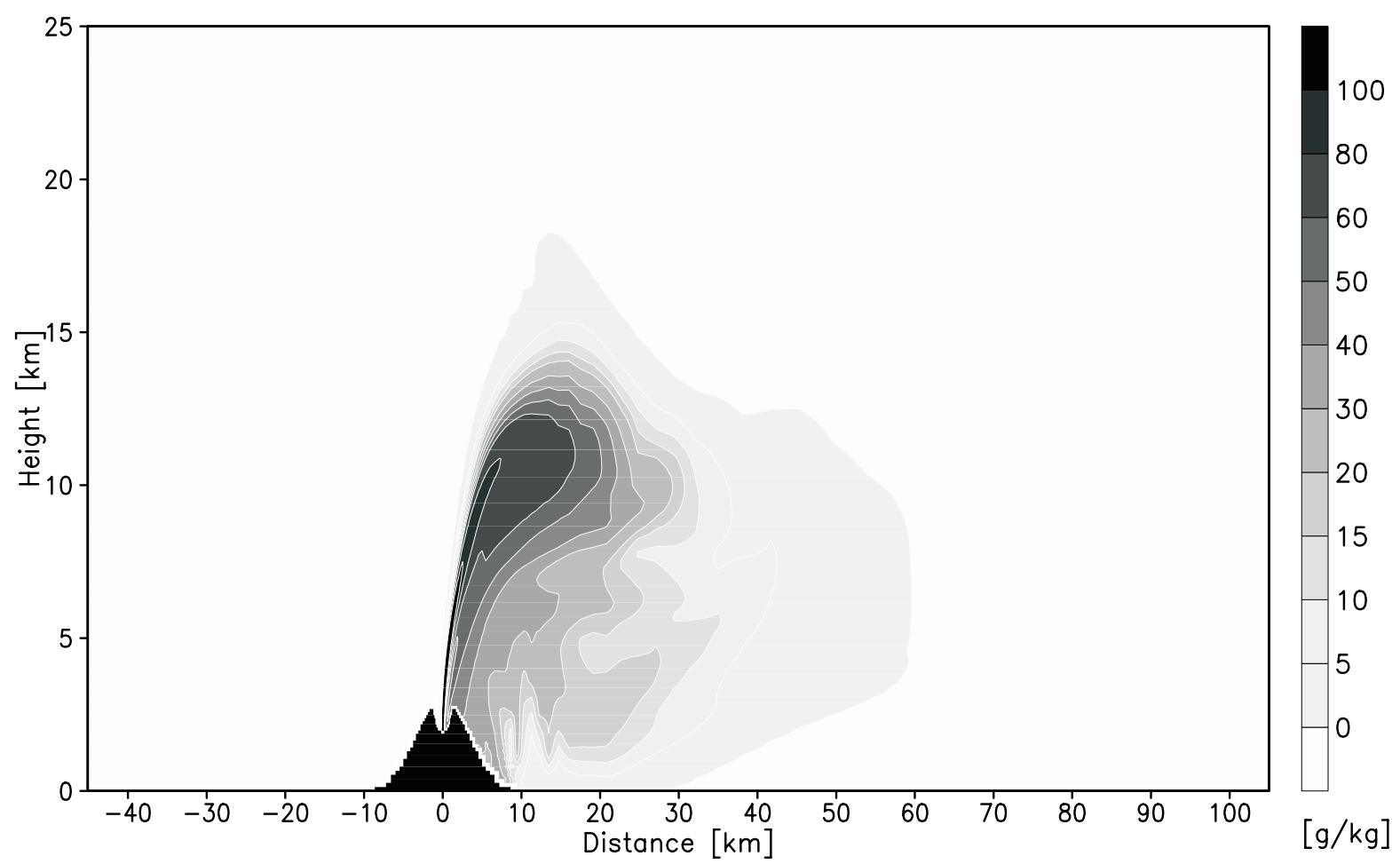

Fig. 15. Specific concentration of lapilli for an experiment with tropical atmospheric background conditions after 25 min. 
profiles become significantly different later. The onset of condensation creates further buoyancy that lifts the plume $15 \%$ higher than without condensation processes. A detailed discussion of the importance of the microphysics can be found in the work of Herzog et al. (1998).

The generation of turbulence and its influence on the dynamics of a volcanic plume is a further phenomenon that is considered in this model. Figs. 10 and 11 show the horizontal and vertical turbulent exchange coefficient for the experiment CYL3. At the edge of the upwind area the strong horizontal shear of the vertical flow generates strong vertical turbulent energy, which is then directly related to the vertical component of the turbulent exchange coefficient. The vertical component of the energy is redistributed in the horizontal direction. Typical values for $K^{q}$ are in the range of $1000 \mathrm{~m}^{2} \mathrm{~s}^{-1}$. Together with the typical length scale for the plume flank of $100 \mathrm{~m}$ this results in a mixing time scale of $10 \mathrm{~s}$ or an equivalent entrainment rate of $10 \mathrm{~ms}^{-1}$, which is the ratio between the diffusion coefficient and the grid size. Fig. 12 shows the potential density for the same situation as that seen in Fig. 7. In accordance with the distribution of the vertical component of the turbulent exchange coefficient, the potential density indicates that the vertical component of turbulent kinetic energy is dissipated inside the plume while at the edge mixing, of the hot particle-laden plume with cold air lets the margin of the plume become lighter and thus unstably stratified.

A further simulation with idealized boundary conditions is performed with cylindrical coordinates and compared with experiment CYL3. This is equivalent to comparing a fissure eruption with infinite extent in the unresolved $y$-axis with an axially symmetric three-dimensional experiment. The comparison gives a concrete indication of the differences between three-dimensional and two-dimensional simulations that cannot use cylindrical coordinates when the required rotational symmetry contradicts with the use of a mean background wind. Fig. 13 shows that in

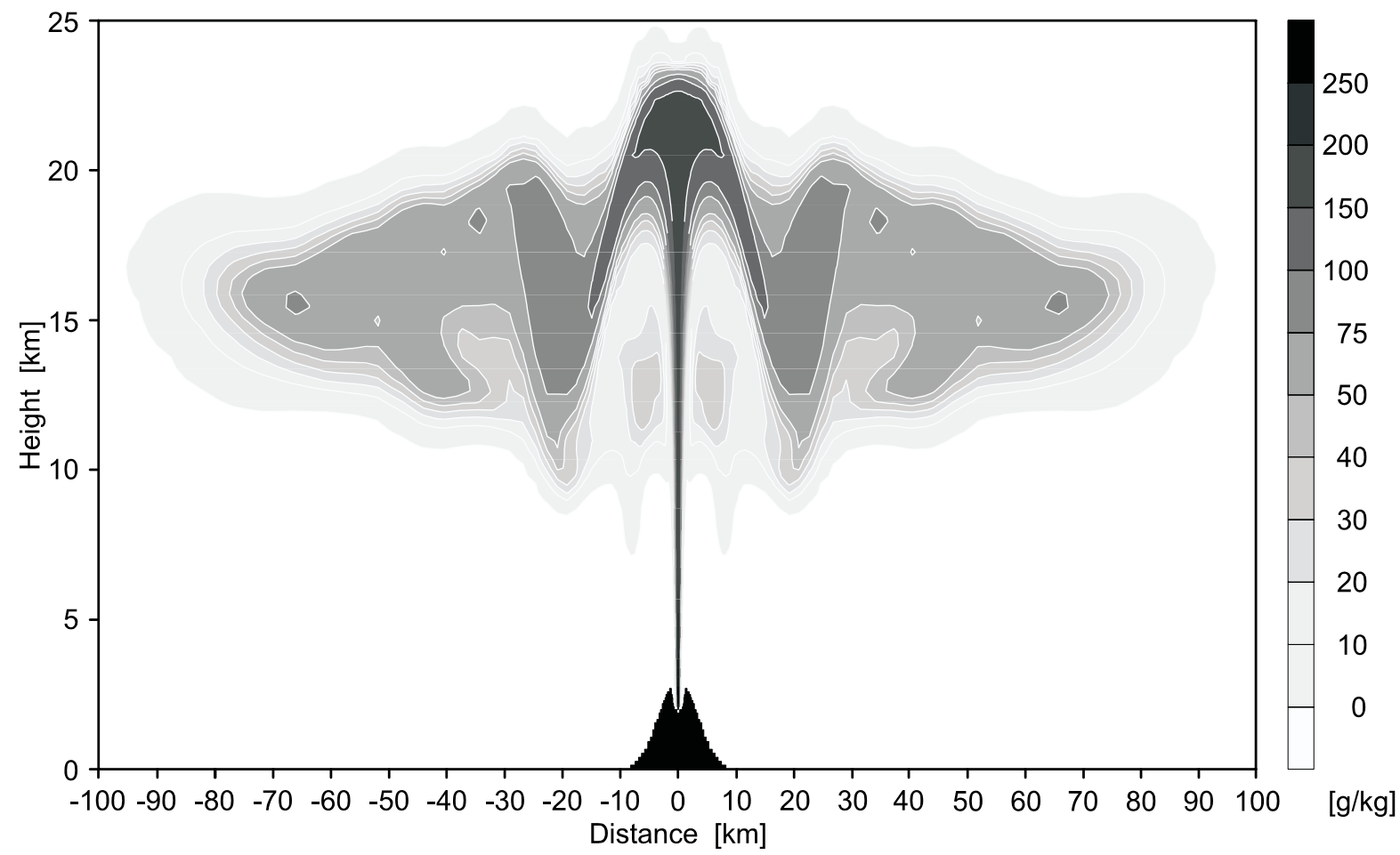

Fig. 16. Specific concentration of ash for an experiment with the same tropical atmospheric background conditions as Fig. 14 but with no background wind after $25 \mathrm{~min}$. 


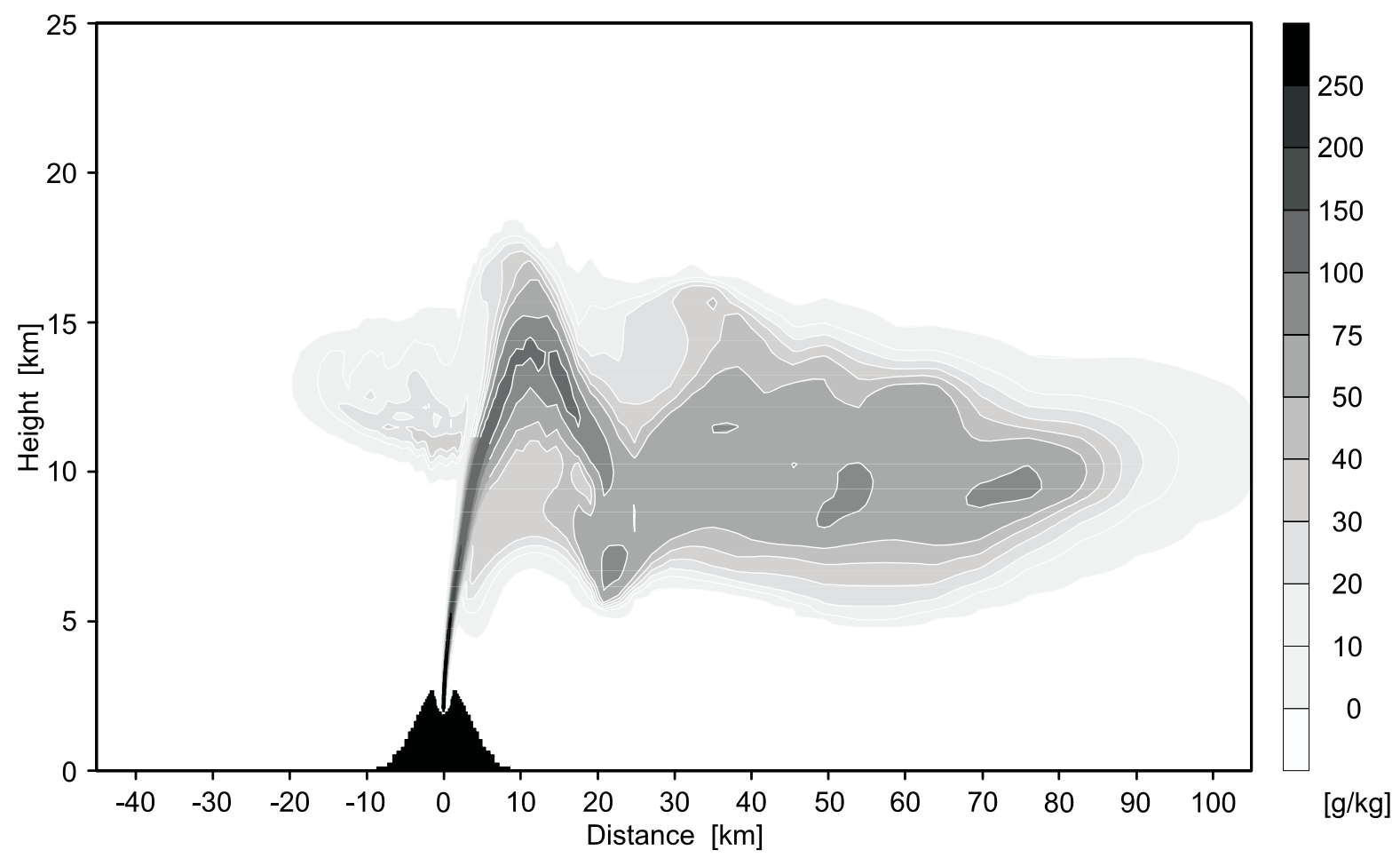

Fig. 17. Specific concentration of ash for an experiment similar to Fig. 14 with tropical wind profile, but with mid-latitude atmospheric background conditions after $25 \mathrm{~min}$.

the case of cylindrical coordinates the plume diverges more quickly in the horizontal direction. This is because in a three-dimensional model, the plume has a further dimension in which to spread out, and thus the temperature anomaly and the concentration of particles decay more rapidly with the horizontal distance from the crater. The same argument holds for turbulent mixing.

\subsection{Dependence on atmospheric background state}

For the following simulations and the forcing parameters the CYL3 grid experiment is used. In addition to the atmospheric profiles for the background temperature and humidity, the background flow is used as well. For the tropical case and with relatively weak winds near the surface, an ash plume (Fig. 14) develops and drifts with the wind to the right. After $25 \mathrm{~min}$ the plume has reached the tropopause at an altitude of $17 \mathrm{~km}$. The same situation in Fig. 15 shows the concentration of lapilli with pronounced downwind sedimentation. For zero-background wind Fig. 16 demonstrates (see also Fig. 14) the impact of the wind profile on the plume height. Finally, Fig. 17 shows the same situation as in Fig. 14 , this time using a mid-latitude atmospheric background profile (McClatchey et al., 1972) and, as for Fig. 14, the tropical wind profile. The plume now overshoots the tropopause level more than in the tropical case with a final downstream plume height that is around the lower tropopause level at an altitude of $10 \mathrm{~km}$, however, more ash is left in the stratosphere.

\section{Discussion and conclusions}

Based on the assumption that all particles are small, a thermo-hydrodynamic model with active tracers has been developed. It is capable to efficiently simulate the development of a volcanic plume on time scales of seconds to hours, on spatial scales 
of a hundred meters to hundreds of kilometers in the horizontal and a hundred kilometers in the vertical. The model solves the full equations of motion for the volume mean momentum and volume mean heat content. All tracer concentrations enter the equation of state for the volume mean density. Tracers used so far are ash and lapilli for the spectrum of silicate particles and various tracers for the water and ice phases that are needed for the cloudphysics.

This initial paper describes the concept and presents a few selected examples for idealized eruptions in order to compare with the results obtained by Valentine and Wohletz (1989), Dobran et al. (1993) and Neri and Macedonio (1996). Further experiments demonstrate that solutions are remarkably different, depending on wether cylindrical coordinates are employed or fissure eruptions are performed. It is also demonstrated that the simulated eddy exchange coefficients, which are computed via prognostic equations for turbulent energy and length scale, and various atmospheric background conditions, control the development of a volcanic plume. Overall, the model appears to capture the development of a plume realistically, although we have not yet compared our model with observational data.

The dynamic equilibrium approach is mostly valid for typical applications of the model, which are characterized by focusing on the large-scale behaviour of a volcanic plume. On the small-scale end of the spectrum of possible applications, however, depressurization in the neighbourhood of a crater, for instance, yields accelerations and thus a pronounced disequilibrium in all spatial directions. The assumption of thermodynamic equilibrium is not strictly valid for bigger particles like lapilli. However, by adding a further heat content equation for lapilli in the style of Valentine and Wohletz (1989) and by replacing the prognostic equation for the total heat with an equation for total heat minus that of lapilli, heat exchanges could be correctly resolved. Then, it is possible to remove model deficiencies that are due to incomplete heat exchange between the fluid's constituents without increasing computer resources significantly.

Basically, a study is required for quantifying errors that arise due to the use of the approximation of dynamic and thermodynamic equilibrium. Such a comparison between solutions using these approxi- mations and using the full set of prognostic equations must be based on the same model in order to exclude any effect due to different numerical implementations.

Another caveat is that the model's concept is based on an advective form for the transport of total heat. The use of transport equations in flux form has failed so far due to numerical null-modes that required too much additional numerical diffusion.

In a companion paper by Herzog et al. (1998) the microphysics is described. In a subsequent paper by Textor et al. (1998) the dissolution processes of volcanic gases into the water phase of a plume will be discussed.

\section{Acknowledgements}

We would like to thank the Volkswagen-foundation for substantially supporting the development of ATHAM under grant EVA (Emission of Volatiles to the Atmosphere). We also acknowledge the motivation through our directors who supported this work despite the fact that the involved institutions have no tradition in volcanic modelling. Finally, we are grateful for the useful comments by Augusto Neri and another unknown reviewer.

\section{Appendix A. Prognostic pressure equation}

In order to present the strategy to solve our model equations with an implicit time-integration scheme, the momentum and pressure equations are written in non-dimensional form:

$$
\begin{aligned}
& \frac{\partial}{\partial t} u=-\frac{\partial}{\partial x} p \\
& \frac{\partial}{\partial t} p=-\frac{\partial}{\partial x} u .
\end{aligned}
$$

If the pressure gradient and the flux divergence are equally weighed in time and the finite-differencing representation in space is:

$$
\begin{aligned}
& u^{l+1}+\frac{\Delta t}{2} \Gamma D_{x}^{\mathrm{s}} p^{l+1}=u^{l}-\frac{\Delta t}{2} \Gamma D_{x}^{\mathrm{s}} p^{l} \\
& p^{l+1}+\frac{\Delta t}{2} D_{x}^{v} u^{l+1}=p^{l}-\frac{\Delta t}{2} D_{x}^{v} u^{l}
\end{aligned}
$$


where $D_{x}^{\mathrm{s}}$ and $D_{x}^{v}$ denote the algebraic representations of the gradient on vector points and divergence on scalar points, respectively, $\Delta t$ is the time step and the superscripts $l$ and $l+1$ mark the old and new time levels. The boundary conditions are introduced via a flag $\Gamma$ that is $\Gamma=0$ for bottom and $\Gamma=1$ for the atmosphere (see also Section 2.7.4). This implies no-slip boundary conditions at the earth's surface. Because Eqs. (34) and (35) are interconnected by the pressure gradient and the flux divergence, one of the two options is to remove $u^{l+1}$ in the pressure equation. This results in an equation for the pressure $p^{l+1}$ only at the new time level:

$$
\begin{aligned}
p^{l+1} & -\frac{\Delta t^{2}}{4} D_{x}^{v}\left(\Gamma D_{x}^{\mathrm{s}} p^{l+1}\right) \\
& =p^{l}-\frac{\Delta t}{2} D_{x}^{v} u^{l}+\frac{\Delta t^{2}}{4} D_{x, v}\left(\Gamma D_{x}^{\mathrm{s}} p^{l}\right)
\end{aligned}
$$

After having found the solution for $p^{l+1}$, is used to calculate the flux $u^{l+1}$ at the new time level. This simple strategy to obtain a stable solution for any chosen time step is used to derive a wave equation for the model's equations for the volume mean momentum and the gas pressure.

\section{Appendix B. Pressure boundary condition}

A subject of controversy is often the use of an elliptic equation for predicting the pressure. It must be stressed that the analytical derivation of Eq. (21) after eliminating the velocities according to Section 2.7.2 and using Eq. (3) does not automatically provide the correct pressure boundary condition. In order to obtain a correct discretization for pressure points near the boundary, first, a no-slip boundary condition is applied for the momentum equation (Eq. (3)) and a no-flux condition for the continuity equation (Eq. (19)). So far, there is no difference to explicit time-stepping models. The discrete prognostic equations are then modified such that specific terms are labelled with the time level index $l+1$ rather than the old time level $l$. Then, the prognostic equation for the pressure $p^{l+1}$ at the new time level is obtained using algebraic manipulations, which do not change the solution at all and thus do not introduce inconsistencies in boundary conditions. This way of deriving a discrete equation for the pressure provides the algebraic representation of the prognostic pressure equation near the boundary. This equation replaces otherwise necessary and mostly intuitive diagnostic pressure boundary conditions that might lead to erroneous physical behaviour. In analogy to the derivation of Eq. (38) the derivation of the model's pressure equation on boundary grid points yields a non-elliptic equation which can be solved nevertheless with an appropriate underrelaxation scheme.

\section{References}

Bursik, M.I., Woods, A.W., 1991. Buoyant, superbouyant and collapsing eruption columns. J. Volcanol. Geotherm. Res. 45, 347-350.

Crowley, W.P., 1968. Numerical advection experiments. Mon. Weath. Rev. 96, 1-11.

Daly, B.J., Harlow, F.H., 1970. Transport equations in turbulence. Physics of Fluids 13, 2634-2649.

Deardorff, J.W., 1980. Stratocumulus-capped mixed layers derived from a three-dimensional model. Boundary-Layer Meteorology 18, 495-527.

Dobran, F., Neri, A., Macedonio, G., 1993. Numerical simulation of collapsing volcanic columns. J. Geophys. Res. 98, 42314259.

Gibson, J.K., Kallberg, P., Uppala, S., Nomura, A., Hernandez, A., Serrano, E., 1997. ERA description. ECMWF re-analysis project report series, Report No. 1, ECMWF, UK.

Glaze, L.S., Baloga, S.M., 1996. The sensitivity of the buoyant plume heights to ambient atmospheric conditions: implications for volcanic eruption columns. J. Geophys. Res. 101, 15291540 .

Glaze, L.S., Baloga, S.M., Wilson, L., 1997. Transport of atmospheric water vapor by volcanic eruption columns. J. Geophys. Res. 102, 6099-6108.

Herzog, M., Graf, H.-F., Oberhuber, J.M., Textor, C., 1998. The effect of phase changes of water on the development of volcanic plumes. J. Volcanol. Geotherm. Res. 87, 55-74.

Kolmogorov, A.N., 1941. The local structure of turbulence in incompressible viscous fluids for very large Reynolds numbers. C.R. Acad. Sci. U.S.S.R. 30, 301-305.

Kwizak, M., Robert, A.J., 1971. A semi-implicit scheme for a grid-point atmospheric model of the primitive equations. Mon. Weath. Rev. 99, 32-36.

Lautenschlager, M., 1983. Parameterisierung der turbulenten Diffusion in der Atmosphäre unter Benutzung der turbulenten Energiegleichung. Technical Report 83/E/60, GKSS Geesthacht, Germany.

Lautenschlager, M., 1985. Initialization of the Euler model MODIS 
with field data from the EPRI plume model validation project. Technical Report 85/E/18, GKSS Geesthacht, Germany.

Lewellen, W.S., 1977. Use of invariant modelling. In: Frost, W., Molden, T.H. (Ed.), Handbook of Turbulence. Plenum, New York, pp. 237-290.

Luther, M., 1986. Advanced methods for steady problems-direct elliptic solvers. In: O'Brien, J.J. (Ed.), Advanced Physical Oceanographic Numerical Modelling. Reidel, Dordrecht, pp. 73-86.

McClatchey, R.A., Fenn, R.W., Selby, J.E.A., Volz, F.E., Garing, J.S., 1972. Optical properties of the atmosphere, 3rd edn., Environmental Research Papers, No. 411.

Mellor, G.L., Yamada, T., 1974. A hierarchy of turbulence closure models for planetary boundary layers. J. Atmos. Sci. 31, 1791-1806.

Mesinger, F., Arakawa, A., 1976. Numerical methods used in atmospheric models. GARP Publications Series No. 17, Vol. 1, pp. 1-64, WMO.

Neri, A., Macedonio, G., 1996. Numerical simulation of collapsing volcanic columns with particles of two sizes. J. Geophys. Res. 101, 8153-8174.

Oberhuber, J.M., 1993. Simulation of the Atlantic circulation with a coupled sea ice-mixed layer-isopycnal general circulation model: Part I. Model description. J. Phys. Oceanogr. 23, 808-829.

O'Brien, J.J. (Ed.), 1986. The steady problem. In: O'Brien, J.J. (Ed.), Advanced Physical Oceanographic Numerical Modelling, Reidel, Dordrecht, pp. 51-72.

Prandtl, L., Wieghardt, K., 1945. Über ein neues Formelsystem für die ausgebildete Turbulenz. Nachr. Akad. Wiss. Göttingen Math. Phys. K 1, 6-19.

Settle, M., 1978. Volcanic eruption clouds and the thermal power output of explosive eruptions. J. Volcanol. Geotherm. Res. 3, 309-324.

Smolarkiewicz, P.K., 1984. A fully multidimensional positive definite advection scheme with small implicit diffusion. J. Comp. Phys. 54, 325-362.

Smolarkiewicz, P.K., 1985. On the accuracy of the Crowley advection scheme. Mon. Weath. Rev. 113, 1425-1429.

Sparks, R.S.J., Wilson, L., 1976. A model for the formation of ignimbrite by gravitational column collapse. J. Geol. Soc. London 132, 441-452.

Textor, C., Graf, H.-F., Herzog, M., Oberhuber, J.M., 1998. On the scavenging of volcanic gases in the eruption column. Submitted to J. Geophys. Res.

Valentine, G.A., Wohletz, K.H., 1989. Numerical models of Plinian eruption columns and pyroclastic flows. J. Geophys. Res. 94, 1867-1887.

Walker, G.P., Wilson, L., Bowell, E.L.G., 1971. Explosive volcanic eruptions: I. The rate of fall of pyroclasts. Geophys. J. R. Astron. Soc. 22, 377-383.

Wilson, L., Sparks, R.S.J., Huang, C.T., Watkins, N.D., 1978. The control of volcanic column heights by eruption energetics and dynamics. J. Geophys. Res. 83, 1829-1836.

Wohletz, K.H., Getchin, T.R., Sanford II, M.T., Jones, E.M., 1984. Hydrodynamic aspects of caldera-forming eruptions: numerical models. J. Geophys. Res. 89, 8269-8285.

Woods, A.W., 1988. The fluid dynamics and thermodynamics of eruption columns. Bull. Volcanol. 50, 169-193.

Woods, A.W., 1993. Moist convection and the injection of volcanic ash into the atmosphere. J. Geophys. Res. 98, 1762717636.

Woods, A.W., 1995. The dynamics of explosive volcanic eruptions. Rev. Geophys. 33, 495-530.

Woods, A.W., Bower, S., 1995. The decompression of volcanic jets in craters. Earth Planet. Sci. Lett. 131, 189-205.

Woods, A.W., Bursik, M.I., 1991. Particle fallout, thermal disequilibrium and volcanic plumes. Bull. Volcanol. 53, 559-570.

Yu, T.W., 1976. Numerical studies of the atmospheric boundary layer with a turbulent energy closure scheme. AMS Third Symposium on Atmospheric Turbulence, Diffusion and Air Qualy, Rayleigh, NC.

Zeman, O., 1990. Dilatation dissipation: the concept and application in modelling compressible mixing layers. Physics of Fluids 2, 178-188.

Zeman, O., 1992. Similarity in supersonic mixing layers. AIAA J. 30, 1277-1283.

Zilitinkevich, S.S., Laikhtman, D.L., Monin, A.S., 1967. Dynamics of the atmospheric boundary layer. Izv. Atmosph. and Oceanic Phys. 3, 170-191. 